\title{
Efficient Algorithms for Finding Optimal Meeting Point on Road Networks
}

\author{
Da Yan, Zhou Zhao and Wilfred $\mathrm{Ng}$ \\ The Hong Kong University of Science and Technology \\ Clear Water Bay, Kowloon, Hong Kong \\ \{yanda, zhaozhou, wilfred\}@cse.ust.hk
}

\begin{abstract}
Given a set of points $Q$ on a road network, an optimal meeting point (OMP) query returns the point on a road network $G=(V, E)$ with the smallest sum of network distances to all the points in $Q$. This problem has many real world applications, such as minimizing the total travel cost for a group of people who want to find a location for gathering. While this problem has been well studied in the Euclidean space, the recently proposed state-of-the-art algorithm for solving this problem in the context of road networks is still not efficient. In this paper, we propose a new baseline algorithm for the OMP query, which reduces the search space from $|Q| \cdot|E|$ to $|V|+|Q|$. We also present two effective pruning techniques that further accelerate the baseline algorithm. Finally, in order to support spatial applications that involve large flow of queries and require fast response, an extremely efficient algorithm is proposed to find a high-quality near-optimal meeting point, which is orders of magnitude faster than the exact OMP algorithms. Extensive experiments are conducted to verify the efficiency of our algorithms.
\end{abstract}

\section{INTRODUCTION}

Applications ranging from location-based services to computer games require optimal meeting point (OMP) query as a basic operation. For example, a travel agency may issue this query to decide the location for a tourist bus to pick up the tourists, so that the tourists can make the least effort to get to the meeting point. This is also true for numerous other scenarios such as an organization that wants to find a place for its members to hold a conference. In strategy games like WorldofWarcraft, a computer player may need this query as part of the artificial intelligence program, to decide the routes of its warriors.

There are two popular ways to define the OMP of a set of points $Q=\left\{q_{1}, q_{2}, \ldots, q_{n}\right\}$, based on two commonly used cost functions:

- min-sum: Find the point $\bar{x}=\arg \min _{x} \sum_{i} d\left(q_{i}, x\right)$, and

- min-max: Find the point $\bar{x}=\arg \min _{x} \max _{i} d\left(q_{i}, x\right)$,

where $d\left(p_{1}, p_{2}\right)$ is the distance between the points $p_{1}$ and $p_{2}$. The metric of distance can be the Euclidean distance (for a Euclidean

Permission to make digital or hard copies of all or part of this work for personal or classroom use is granted without fee provided that copies are not made or distributed for profit or commercial advantage and that copies bear this notice and the full citation on the first page. To copy otherwise, to republish, to post on servers or to redistribute to lists, requires prior specific permission and/or a fee. Articles from this volume were invited to present their results at The 37th International Conference on Very Large Data Bases, August 29th - September 3rd 2011, Seattle, Washington.

Proceedings of the VLDB Endowment, Vol. 4, No. 11

Copyright 2011 VLDB Endowment 2150-8097/11/08... \$ 10.00. space) or the network distance (for a road network). The network distance between two points on a road network is the length of the shortest path connecting them. Figure 1(a) illustrates the idea of OMPs using a road network with six people at the six black points, who want to meet together at some location on the road network. The upward (left) triangle in Figure 1(a) is the min-max OMP, and the downward (right) one is the min-sum OMP.

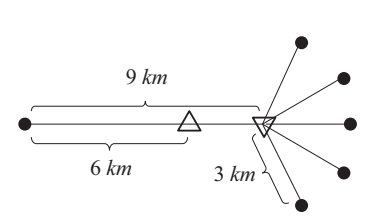

(a) Optimal Meeting Points

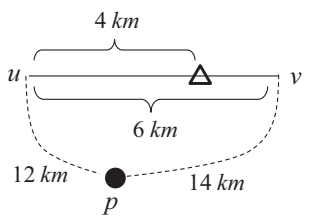

(b) Split Point
Figure 1: Example of OMPs and Split Points.

A min-sum OMP minimizes the total travel distance of all the people, while a min-max OMP minimizes the elapsed travel time. For the example in Figure 1(a), the person at the black point on the left has to walk for $9 \mathrm{~km}$ to reach the min-sum OMP, and those on the right have to wait for him after they reach the meeting point. On the other hand, all the people will walk for $6 \mathrm{~km}$ to get to the minmax OMP, which is faster than the min-sum one. Note that both types of OMPs may not be unique in general. For example, for two people at two different locations on a road network, the min-sum OMP could be anywhere on the shortest path between them.

As transportation is getting more and more convenient nowadays, min-sum OMPs are often preferred over min-max OMPs. Consider a multi-national corporation that plans to hold a meeting to let all its executive officers in China report to its CEO from the headquarter in USA. The ideal location for the meeting is in China since most of the participants are in China, while the min-max OMP may be within some European country on the path between USA and China. If the meeting is held in China, only the CEO from USA needs to set out earlier to fly to the meeting location, while the other participants can set out at a later time, and the travel cost is minimized. On the other hand, if the meeting is held in the min-max OMP, all the participants have to set out early and the total travel cost is huge. Therefore, we study the min-sum OMP query in this paper, and whenever OMP (or optimal meeting point) is mentioned later, we are referring to the min-sum OMP.

While the OMP query has been extensively studied in the Euclidean space, the state-of-the-art algorithm of processing the query in road networks is still not efficient. In this paper, we identify an interesting property of this problem, which greatly prunes the search space compared with the best-known technique. Two effec- 
Table 1: Summary of Notations.

\begin{tabular}{|c|l|}
\hline Notation & \multicolumn{1}{c|}{ Meaning } \\
\hline$\overline{p_{1}, p_{2}}$ & the shortest path between points $p_{1}$ and $p_{2}$ \\
\hline$p_{1} \sim p_{2}$ & $\begin{array}{l}\text { the line segment of an edge with endpoints } p_{1} \\
\text { and } p_{2} \quad\left(p_{1} \text { and } p_{2} \text { are on the same edge }\right)\end{array}$ \\
\hline$d(\ell)$ & the length of the path/segment $\ell$ \\
\hline$s d(p, Q)$ & $\begin{array}{l}\text { the sum of distances of point } p \text { to the points in } \\
\text { query set } Q \quad(Q \text { is omitted when it is clear from } \\
\text { the context) }\end{array}$ \\
\hline
\end{tabular}

tive pruning rules are proposed to further accelerate query processing. Finally, in order to support spatial applications that require fast response, we propose another algorithm to find a high-quality near-optimal meeting point in considerably less time.

The rest of this paper is organized as follows: Section 2 reviews the previous studies that are highly relevant to the OMP query. Then, we introduce our efficient algorithms, describe the underlying idea, and analyze the time complexity in Section 3. Extensive experiments are presented in Section 4 to show the efficiency of our algorithms for the OMP queries. Finally, we conclude our paper in Section 5 .

Table 1 summarizes the notations used throughout this paper.

\section{RELATED WORK}

Like the window query [1] and the various nearest neighbor queries $[9,11,6,12,5]$, the OMP query is also fundamental in spatial databases. The studies of min-sum OMP query in the context of Euclidean space date back to the $60 \mathrm{~s}-70$ s $[7,8,4,3]$. When the Euclidean distance is adopted as the metric of distance, the OMP query is called the Weber problem [7], and the OMP is called the geometric median of the query point set $Q$.

Cooper [7] extended the Weber problem by posing the problem of minimizing the weighted sums of powers of the Euclidean distances, which was further generalized to handle radial cost functions by Reuven Chen [4]. However, it has been shown that no closed form formula exists for the Weber problem and its generalizations, and these problems are usually solved by gradient descent methods, with initial point chosen as the center of gravity of the query point set $Q$. Fortunately, the sum of Euclidean distances is a convex function, since it is the composite of linear-norm-sum functions, all of which preserve convexity [2]. As a result, the gradient descent method is able to approach the global minimum without the worry of being stuck at local minimal values.

On the other hand, the OMP query is not well explored in terms of road networks, where the network distance is adopted as the distance metric. However, compared with the Weber problem, this is a more realistic scenario for location-based services. Recently, [19] proposed a solution to this problem by checking all the split points on the road network. For a point $p$ on a road network, its split point on edge $(u, v)$ is defined to be the point $x$ such that $d(\overline{p, u})+d(u \sim x)=d(\overline{p, v})+d(v \sim x)$ (see Table 1 for the notations). Figure 1(b) illustrates the idea of split point, where the dotted curves denote the shortest paths between the end points. The location marked by the triangle in Figure 1(b) is the split point $x$ of $p$ on edge $(u, v)$. The shortest path from $p$ to any point on the left (or right) of $x$ on edge $(u, v)$ passes through $u$ (or $v$ ).

It is proved in [19] that an OMP must exist among the split points, which leads to an algorithm that checks the split point of each query point in $Q$ on each edge in the road network $G=$ $(V, E)$, and picks the split point with the smallest sum of network distances as the OMP. As a result, the search space is $|Q| \cdot|E|$, which is huge. Although [19] includes a pruning technique to skip some split points that are guaranteed not to be an OMP, the search space after pruning is still very large. Therefore, a novel road network partitioning scheme is proposed in [19] to further prune the search space, based on the property that the OMP is strictly confined within the partition where all the objects in the query set $Q$ are located. This leads to the algorithm which first obtains the smallest partition that encloses all the basic network partitions where the points in $Q$ belong, and then checks the split points in this partition.

A highly relevant but different type of query is the group nearest neighbor query $[13,20]$. Given two sets of points $P$ and $Q$, a group nearest neighbor (GNN) query retrieves the point(s) of $P$ with the smallest sum of distances to all the points in $Q$. GNN queries can be applied, for instance, when $n$ users at locations $Q=$ $\left\{q_{1}, q_{2}, \ldots, q_{n}\right\}$ want to choose a restaurant to have dinner together, among a set of restaurants at locations $P=\left\{p_{1}, p_{2}, \ldots, p_{m}\right\}$ in the city. The GNN query is different from the OMP query in that the candidate result locations of the former is the set $P$ while the candidate result locations of the latter is all the possible locations on the road network. Therefore, the OMP query is more difficult than the GNN query due to its infinite search space. The OMP query is also more general than the GNN query in that it does not require users to determine the kind of place to meet at in advance. For a travel agency that needs to decide the location for a tourist bus to pick up the tourists, the set $P$ does not even exist.

\section{ALGORITHM}

It is proved in [19] that an OMP must exist among the split points. As a result, [19] proposed to check all the $|Q| \cdot|E|$ split points for query set $Q$ on the road network $G=(V, E)$, and to pick the one with the smallest sum of distances to all the points in $Q$ as the OMP. However, the $|Q| \cdot|E|$ search space is still very huge. In Section 3.1, we improve the search space to $|V|+|Q|$ by proving that $V \cup Q$ must contain an OMP, and propose our baseline algorithm that only checks all the vertices in $V$ and all the points in $Q$, and picks the one with the smallest sum of distances to all the points in $Q$ as the OMP.

Then, in Section 3.2, we improve the performance of our baseline algorithm by two online convex-hull-based pruning techniques, which restrains the search space to a small region of the whole road network. This region is always smaller than the partition obtained by [19] that uses the off-line road network partitioning scheme. As a result, our pruning technique achieves better pruning effect.

To further support spatial applications that involve simultaneous evaluation over many queries and require fast response, we propose another algorithm in Section 3.3 that finds a high-quality nearoptimal meeting point in considerably less time.

\subsection{Baseline Algorithm}

For a query point set $Q$, the baseline algorithm of [19] treats all the $|Q| \cdot|E|$ split points in the road network $G=(V, E)$ as candidates for the OMP. However, it is not necessary to compute all the split points and evaluate the sums of distances for all of them. In fact, it is sufficient to consider only the vertices in $V$ and the points in $Q$ for the OMP, which we prove next:

LEMMA 1. Given a query point set $Q$, let $s d(p)$ denote the sum of distances of point $p$ to the points in $Q$. Suppose that no point in $Q$ is on edge $(u, v)$ except for the two end points $u$ and $v$, then for any point $x$ on edge $(u, v)$, we have $s d(x) \geq \min \{s d(u), s d(v)\}$.

Proof. For a point $x$ on edge $(u, v)$, we denote $Q_{u}$ as the set of query points whose shortest paths to $x$ pass through $u$. Accordingly, $Q_{v}=Q-Q_{u}$ is the set of query points whose shortest paths 


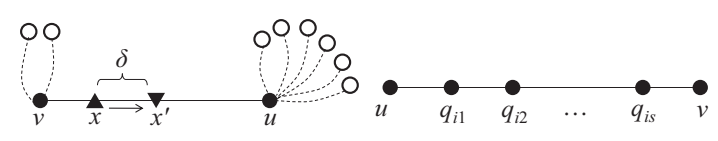

(a)

(b)

Figure 2: Illustration of Lemma 1 and Theorem 1.

to $x$ pass through $v$. Without loss of generality, let us assume that $\left|Q_{u}\right| \geq\left|Q_{v}\right|$. Figure 2(a) illustrates this scenario, where the hollow points are the query points and the dotted lines are part of their shortest paths to $x$.

Now consider the point $x^{\prime}$ on edge $(u, v)$ which is $\delta$ closer to $u$ than $x$. Let $Q_{a b}(a, b \in\{u, v\})$ denote the set of query points that belong to $Q_{a}$ when the meeting point is $x$ and belong to $Q_{b}$ when the meeting point is $x^{\prime}$. Therefore, we can classify the points in $Q$ into four disjoint sets: $Q_{u u}, Q_{v v}, Q_{u v}$ and $Q_{v u}$.

For these four point sets, we have the following properties:

- $\forall p \in Q_{u u}, d\left(\overline{p, x^{\prime}}\right)=d(\overline{p, x})-\delta$.

Proof: $d\left(\overline{p, x^{\prime}}\right)=d(\overline{p, u})+d\left(u \sim x^{\prime}\right)=d(\overline{p, u})+[d(u \sim$ $x)-\delta]=[d(\overline{p, u})+d(u \sim x)]-\delta=d(\overline{p, x})-\delta$.

- $\forall p \in Q_{v v}, d\left(\overline{p, x^{\prime}}\right)=d(\overline{p, x})+\delta$.

Proof: $d\left(\overline{p, x^{\prime}}\right)=d(\overline{p, v})+d\left(v \sim x^{\prime}\right)=d(\overline{p, v})+[d(v \sim$ $x)+\delta]=[d(\overline{p, v})+d(v \sim x)]+\delta=d(\overline{p, x})+\delta$.

- $Q_{u v}=\emptyset$.

Proof: For any $p \in Q_{u}$ when the meeting point is $x$, we have $d(\overline{p, v})+d\left(v \sim x^{\prime}\right)=d(\overline{p, v})+[d(v \sim x)+\delta]>d(\overline{p, v})+$ $d(v \sim x) \geq d(\overline{p, x})=d(\overline{p, u})+d(u \sim x)=d(\overline{p, u})+$ $\left[d\left(u \sim x^{\prime}\right)+\delta\right]>d(\overline{p, u})+d\left(u \sim x^{\prime}\right)$, which implies that the shortest path from $p$ to $x^{\prime}$ cannot pass through $v$ (i.e. $p \notin Q_{v}$ ) when the meeting point is $x^{\prime}$.

- $\forall p \in Q_{v u}, d\left(\overline{p, x^{\prime}}\right) \leq d(\overline{p, x})+\delta$.

Proof: $d\left(\overline{p, x^{\prime}}\right) \leq d(\overline{p, v})+d\left(v \sim x^{\prime}\right)=d(\overline{p, v})+d(v \sim$ $x)+\delta=d(\overline{p, x})+\delta$.

Therefore, we have

$$
\begin{aligned}
& \sum_{q \in Q} d(\overline{q, x})=\left(\sum_{q \in Q_{u u}}+\sum_{q \in Q_{v v}}+\sum_{q \in Q_{u v}}+\sum_{q \in Q_{v u}}\right) d(\overline{q, x}) \\
\geq & \sum_{q \in Q_{u u}}\left[d\left(\overline{q, x^{\prime}}\right)+\delta\right]+\left(\sum_{q \in Q_{v v}}+\sum_{q \in Q_{v u}}\right)\left[d\left(\overline{q, x^{\prime}}\right)-\delta\right] \\
= & \left(\sum_{q \in Q_{u u}}+\sum_{q \in Q_{v v}}+\sum_{q \in Q_{v u}}\right) d\left(\overline{q, x^{\prime}}\right) \\
& +\delta\left(\left|Q_{u u}\right|-\left|Q_{v v}\right|-\left|Q_{v u}\right|\right)
\end{aligned}
$$

As $Q_{u v}=\emptyset$, we have $\sum_{q \in Q_{u v}} d\left(\overline{q, x^{\prime}}\right)=0$. Besides, since $\left|Q_{u}\right| \geq\left|Q_{v}\right|$ when the meeting point is $x$, i.e. $\left|Q_{u u}\right|+\left|Q_{u v}\right| \geq$ $\left|Q_{v u}\right|+\left|Q_{v v}\right|$, we have $\left|Q_{u u}\right|-\left|Q_{v v}\right|-\left|Q_{v u}\right| \geq-\left|Q_{u v}\right|=\overline{0}$ According to the above analysis,

$$
\begin{aligned}
\sum_{q \in Q} d(\overline{q, x}) & \geq\left(\sum_{q \in Q_{u u}}+\sum_{q \in Q_{v v}}+\sum_{q \in Q_{u v}}+\sum_{q \in Q_{v u}}\right) d\left(\overline{q, x^{\prime}}\right) \\
& =\sum_{q \in Q} d\left(\overline{q, x^{\prime}}\right)
\end{aligned}
$$

Thus, we can conclude that $s d\left(x^{\prime}\right) \leq s d(x)$ for arbitrary $x, x^{\prime}$ and $\delta$. If we set $x^{\prime}$ to be $u$, we reach the conclusion that $\forall x$ on edge $(u, v), s d(u) \leq s d(x)$. Due to the symmetry of $u$ and $v$, if
$\left|Q_{v}\right| \geq\left|Q_{u}\right|$ we get: $\forall x$ on edge $(u, v), s d(v) \leq s d(x)$. To sum up, $\forall x$ on edge $(u, v), \min \{s d(u), s d(v)\} \leq s d(x)$.

Intuitively, Lemma 1 shows that for any edge on the road network, one of the endpoints is at least as good as any other point on the edge in terms of the sum-of-distances value. Now, let us take into consideration the special case where there exist some query points on an edge, as illustrated by Figure 2(b). By using Lemma 1, we have the following theorem:

THEOREM 1. Given an OMP query with query point set $Q$ on a road network $G=(V, E), V \cup Q$ contains an $O M P$.

Proof. For each edge $(u, v)$ that contains some query points on it, but not at the end points $u$ and $v$, let us denote these query points as $q_{i_{1}}, q_{i_{2}}, \ldots, q_{i s}$, as illustrated in Figure 2(b). We introduce $s$ dummy vertices $p_{i_{1}}, p_{i_{2}}, \ldots, p_{i s}$ on the edge $(u, v)$, where each dummy vertex $p_{i j},(j=1,2, \ldots, s)$ is located at $q_{i j}$.

After the introduction of the dummy vertices for all the edges that contain some query points on it but not at its end points, we obtain another road network $G^{\prime}$ such that all the query points in $Q$ are at its vertices. Since the vertex set of $G^{\prime}$ is $V \cup Q$, we can conclude that $V \cup Q$ contains an OMP according to Lemma 1.

Theorem 1 is general enough for road networks of any topology, since its proof (including that of Lemma 1) relies only on the fact that the road network $G$ is a graph. For example, the edge length can refer to the travel delay rather than physical distance. In fact, we can obtain the following more general statement:

THEOREM 2. Given a point set $Q=\left\{q_{1}, q_{2}, \ldots, q_{n}\right\}$ on an arbitrary graph $G=(V, E)$, where each point $q_{i}$ is associated with $a$ weight $w_{i}$. If all the weights are integers or rational numbers, then $V \cup Q$ must contain the point $\bar{x}=\arg \min _{x} \sum_{i} w_{i} \cdot d\left(\overline{q_{i}, x}\right)$.

Proof. See Appendix A.

Note that the idea of Theorem 1 to find the OMP among $V \cup Q$ does not contradict the idea of [19] to find the OMP among the split points. See Appendix B for a detailed discussion on this point.

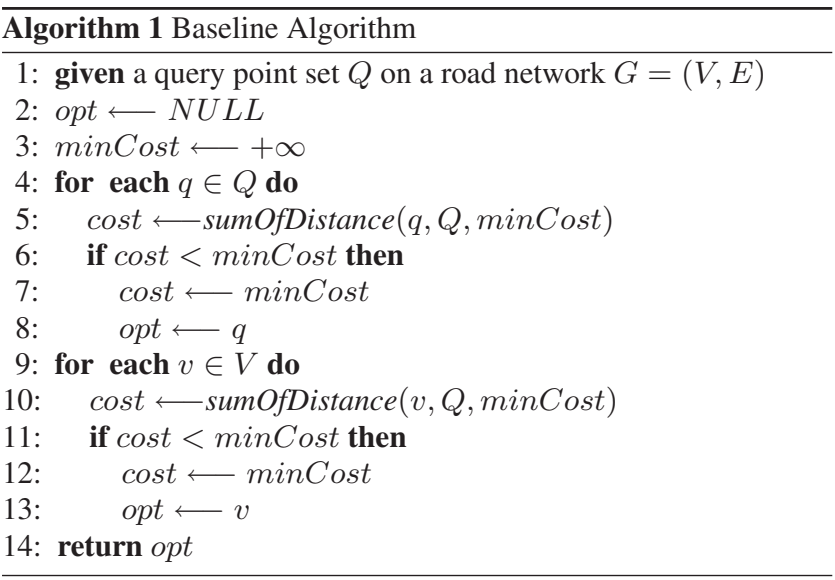

Based on Theorem 1, we design our baseline algorithm (Algorithm 1) to check all the points in $Q$ and all the vertices in $V$, and pick the one with smallest sum of network distances to all the points in $Q$ as the OMP. The function sumOfDistance in Lines 5 and 10 of Algorithm 1 computes the sum of network distances of $q$ (or $v$ ) to all the points in $Q$, which is detailed in Algorithm 2 .

Lines 4-5 in Algorithm 2 return the partially computed value of the sum of distances for $v$ if it is already larger than minCost. Let 


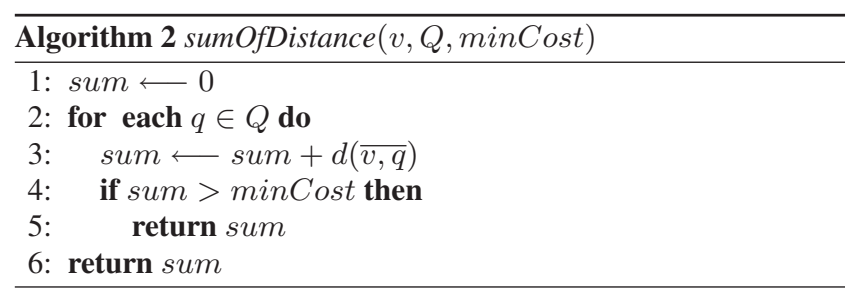

minCost be the smallest sum of distances that is already found for the time being, then Lines $4-5$ act as a pruning step to stop the computation for $v$ since it cannot be the optimal point. Lines 8 and 11 in Algorithm 1 then automatically filter out such points.

A basic operation in all our algorithms is to obtain the length of the shortest path between two points $p_{1}$ and $p_{2}$, i.e. $d\left(\overline{p_{1}, p_{2}}\right)$, (e.g. Line 3 in Algorithm 2). Since shortest path computation is not our focus, we simply run Dijkstra algorithm for each vertex in the road network, and write all the obtained information into a index file on the disk. For the details on the construction and organization of our index file, please refer to Appendix C.

After the shortest path index file is constructed, the length of the shortest path between two vertices $u, v \in V$ on the road network $G=(V, E)$, i.e. $d(\overline{u, v})$, can be obtained by only one I/O operation, and the shortest path $\overline{u, v}=\left(p_{0}=u, p_{1}, p_{2}, \ldots, p_{\ell}=v\right)$ can be obtained by $\ell \mathrm{I} / \mathrm{O}$ operations. Further, we can utilize the above operation to obtain $d(\overline{p, v})$ for any point $p$ on the road network and any vertex $v \in V$ by two I/O operations, and $d\left(\overline{p_{1}, p_{2}}\right)$ for any two points $p_{1}$ and $p_{2}$ on the road network by at most four $\mathrm{I} / \mathrm{O}$ operations. The reasoning of the above statements is presented in Appendix D. To sum up, only $O(1) \mathrm{I} / \mathrm{O}$ operations are required to obtain the the length of the shortest path between two arbitrary points on the road network, while $\ell \mathrm{I} / \mathrm{O}$ operations are required to obtain a shortest path of length $\ell$ between two points.

As there are $|V|$ vertices to check (Lines 9-13 in Algorithm 1), each of which requires $O(|Q|)$ I/O operations to compute the value of the sum of distances (Lines 2 and 3 in Algorithm 2), the total number of I/O operations required is $O(|Q| \cdot|V|)$. Similarly, Lines 4-8 in Algorithm 1 take $|Q| \cdot O(|Q|)=O\left(|Q|^{2}\right)$ I/O operations. To sum up, the time complexity of Algorithm 1 is $O(|Q|$. $\left.|V|+|Q|^{2}\right)$.

\subsection{Pruning Based on Convex Hull}

Compared with the split-point-based method in [19], our baseline algorithm in Section 3.1 has already significantly reduced the search space of the OMP query. However, there is still room for the further pruning of the search space when the edge length of the road networks is based on the physical distance. For example, if all the query points are in California, then there is no need to check the vertices in Utah on the road network. Based on this rationale, [19] cuts the whole road network into partitions, and checks only those split points that are in the smallest partition enclosing all the basic network partitions where the query points belong. Consider the partitioned road network shown in Figure 3(a), where the four black points are the query points, [19] only checks the split points in the gray area.

However, the correctness of that pruning technique relies on the assumption that whenever two roads cross with each other, there is an intersection vertex at the crossing point. This assumption may not be true in reality, e.g. when one road is a viaduct or a tunnel. Appendix E shows an example road network where the pruning technique of [19] makes mistakes.

Furthermore, the pruning effectiveness of that network partitioning scheme is still not sufficient. Referring to Figure 3(a) again, it

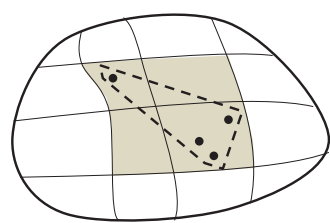

(a) Graph Partitioning

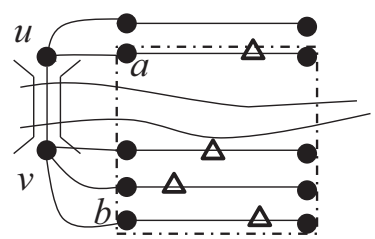

(b) Counterexample of Alg. 3
Figure 3: Illustration of Pruning Techniques.

is intuitive that the optimal meeting point must appear in the region surrounded by the dotted curve, and it is not necessary to check the remaining part of the gray area, which is the area checked by the network partitioning scheme. Besides, there is no strict underlying principle on how to partition a road network mentioned in [19].

Now, we propose two online convex-hull-based pruning techniques that are more effective. Although our online pruning techniques do not rely on a pre-computed index, they provide higher efficiency since the dominating factor of the query processing time is the number of points/vertices to check, and the time of convex hull computation required by our techniques is negligible.

Before describing our pruning techniques, we first define the format of a query point $q$ in the query point set $Q$. Since a query point on a road network $G=(V, E)$ must be on some edge $(u, v) \in E$, we define the format of a query point as follows:

DEFINITION 1. Given a road network $G=(V, E)$ and a query point $q$ on edge $(u, v) \in E$, we define the format of $q$ as the triplet $(u, v, \lambda)$ such that $\overrightarrow{u q}=\lambda \cdot \overrightarrow{u v}$.

According to Definition $1, q$ is at the vertex $u$ when $\lambda=0$, and at $v$ when $\lambda=1$.

The first phase of our pruning techniques is to collect into a set $P$ those end points of all the edges which the query points in $Q$ are on, and then compute the convex hull of the point set $P$. Algorithm 3 details this process, where convexHull $(P)$ computes the convex hull of the point set $P$ using Andrew's Monotone Chain algorithm [15], which takes $O(|P| \log |P|)$ time.

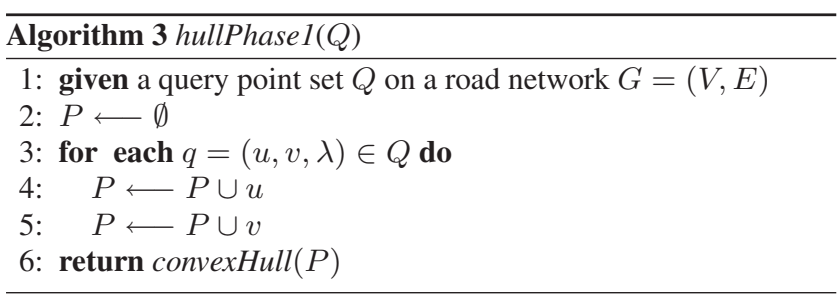

The first phase pruning simply checks the points in $Q$, and the vertices in the region surrounded by the convex hull computed by Algorithm 3 to find the optimal meeting point. However, this is not sufficient, as we are going to illustrate.

Consider the road network in Figure 3(b) where a bridge crosses over a river. The query points are marked by the triangles. The convex hull returned by Algorithm 3 is the one drawn with dotted lines. It is easy to check that $v$ is the OMP, but it is outside of the region surrounded by the convex hull.

In order to avoid such false negatives in search space pruning, we propose a second phase of convex hull computation. Suppose that the convex hull returned by hullPhase $1(Q)$ is represented as $H=\left(h_{1}, h_{2}, \ldots, h_{\ell}, h_{1}\right)$ where the points $h_{i}$ on $H$ are listed in clockwise order, we find the shortest path for each pair of neighboring points on $H$, insert all the points on these paths into a set $S$, 


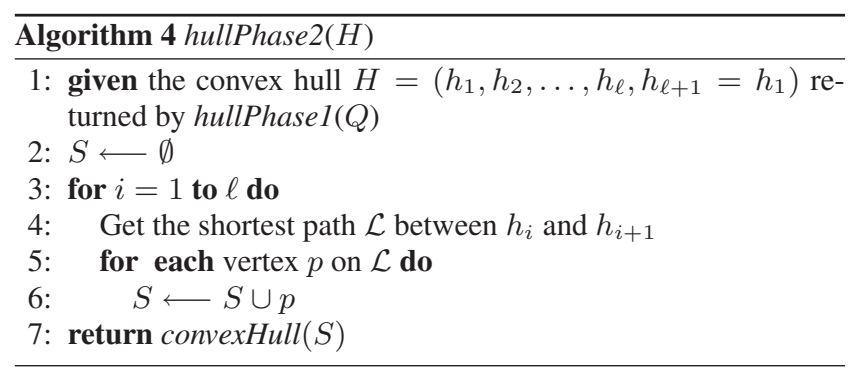

and then compute the convex hull of $S$. The process of the second phase pruning is detailed in Algorithm 4, where we use our index file to obtain the shortest path between two vertices in Line 4.

For the previous example in Figure 3(b), the OMP $v$ is now in the region surrounded by the convex hull returned by Algorithm 4, since it is on the shortest path between $a$ to $b$, which are the two neighboring vertices on the convex hull returned by Algorithm 3 .

Let $|H|$ denote the number of vertices on $H$, and $\left|\mathcal{L}_{\max }\right|$ be the maximum number of points on the shortest path between two neighboring points on $H$, then Algorithm 4 takes $O\left(|H| \cdot\left|\mathcal{L}_{\text {max }}\right|\right.$. $\left.\log \left(|H| \cdot\left|\mathcal{L}_{\max }\right|\right)\right)$ time.

Now, we present Algorithm 5 that first performs our two-phase online convex hull computation, and then checks only the query points and the vertices in the region surrounded by the convex hull to find the OMP.

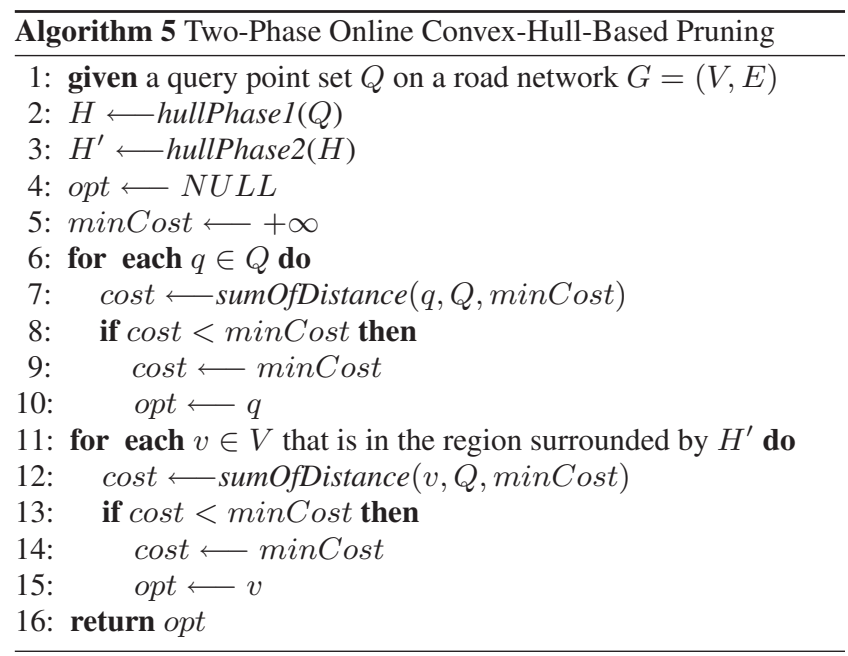

If we use only the first phase of convex hull computation, Lines 2 and 3 in Algorithm 5 can be replaced by " $H^{\prime} \longleftarrow$ hullPhase1(Q)".

To support efficient range query evaluation in Line 11 in Algorithm 5, we organize all the vertices in $V$ by a kd-tree. The query window is the minimum bounding box (MBR) of the convex hull $H^{\prime}$, and for the vertices in the MBR, a refinement step is performed to obtain the vertices that are really in the region surrounded by $H^{\prime}$.

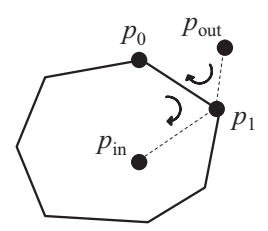

Figure 4: Points Inside/Outside the Convex Hull.
We check whether a vertex is inside the region surrounded by the convex hull using the following property: given three points $p_{1}=\left(x_{1}, y_{1}\right), p_{2}=\left(x_{2}, y_{2}\right)$ and $p_{3}=\left(x_{3}, y_{3}\right)$, let us define $c c w\left(p_{1}, p_{2}, p_{3}\right)=\left(x_{2}-x_{1}\right) *\left(y_{3}-y_{1}\right)-\left(x_{3}-x_{1}\right) *\left(y_{2}-y_{1}\right)$ (or simply ccw). The angle $\overline{p_{1} p_{2} p_{3}}$ is in counter-clockwise order if $c c w>0$, in clockwise order if $c c w<0$, and $p_{1}, p_{2}$ and $p_{3}$ are on the same line if $c c w=0$. To judge whether a point $p$ is inside or on the boundary of the region surrounded by a convex hull $H^{\prime}$, we have the following theorem:

THEOREM 3. A point $p$ is inside or on the boundary of the region surrounded by a convex hull $H^{\prime}$, if and only if for any edge $\left(p_{0}, p_{1}\right)$ on $H^{\prime}$ where $p_{0}$ and $p_{1}$ are listed in clockwise order, ccw $\left(p_{0}\right.$, $\left.p_{1}, p\right) \leq 0$.

Since the result convex hull $H^{\prime}$ returned by Andrew's Monotone Chain algorithm is a stack of points on $H^{\prime}$ that are pushed in counter-clockwise order, for some point $p$, we pop each edge $\left(p_{0}, p_{1}\right)$ on $H^{\prime}$ (in clock-wise order) and check $c c w\left(p_{0}, p_{1}, p\right)$. Figure 4 illustrates the process. As long as we find $c c w>0$ for an edge on $H^{\prime}, p$ is outside the region surrounded by $H^{\prime}$ and thus pruned. Otherwise, we need to check $p$. Let $\left|H^{\prime}\right|$ denote the number of vertices on $H^{\prime}$, then this check takes $O\left(\left|H^{\prime}\right|\right)$ time.

Algorithm 5 is able to find the OMP on almost all real road networks except for very unusual cases as illustrated in Appendix E. Actually, we have done extensive experiments on the road network datasets of 46 states in US [21], and the dataset of "city of Oldenburg" (OL) [22]. Altogether 1700 randomly generated OMP queries are performed on each dataset. Algorithm 5 only fails to return the OMP in 5 of the 79900 queries in total, and the sum-ofdistances values of these result meeting points are all within $0.1 \%$ more than the smallest sum-of-distances value.

\subsection{Fast Greedy Algorithm for OMP Queries}

Although the convexity property of the "sum of Euclidean distances" function no longer holds for the sum of network distances, the breach of this property is not significant since both types of distances are defined over a metric space.

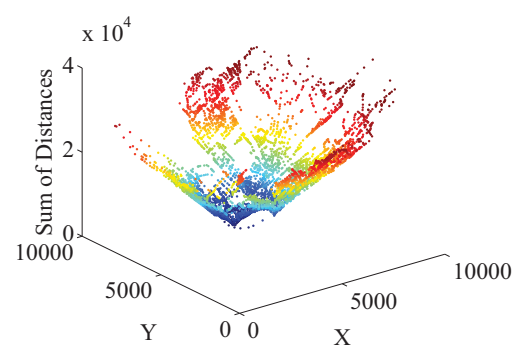

Figure 5: "Sum of Distances" Values at All Network Vertices.

For example, Figure 5 shows the values of the sums of network distances at all the vertices (represented by $(X, Y)$-coordinates) in the road network of the OL dataset for an OMP query. Warmer vertex color represents larger sum-of-distances value. From the shape of the function, we can observe that "convexity" is preserved to a great extent. One can easily obtain the same observation for arbitrary query point sets on road networks. Inspired by this observation and the gradient descent methods of $[7,8]$, we propose a greedy algorithm as shown in Algorithm 6, where we denote the sum-of-distances value of a vertex $u$ as $s d(u)$, and the set of neighboring vertices of $u$ as $N B(u)$. 


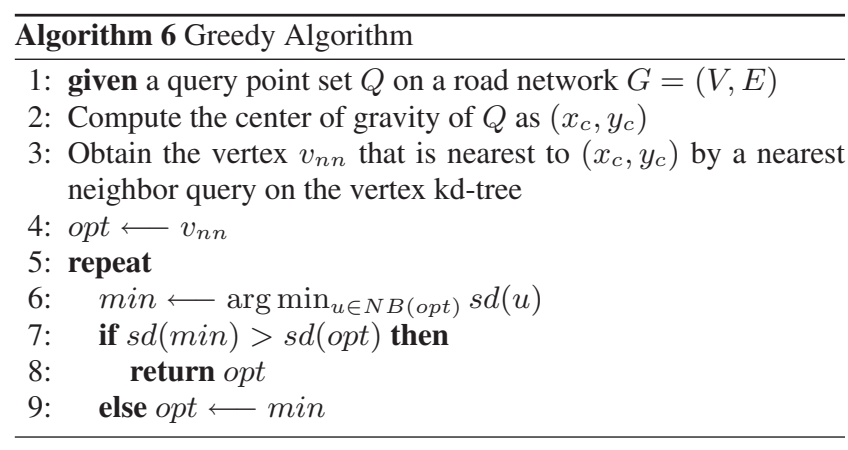

Algorithm 6 first computes the center of gravity $\left(x_{c}, y_{c}\right)$ of the query point set $Q$ (Line 2), which is the common choice of the initial point for the gradient descent methods of the Weber problem. As our initial point should be a vertex rather than an arbitrary point in the 2D space, Algorithm 6 picks the vertex that is closest to $\left(x_{c}, y_{c}\right)$ as the initial point (Line 3 ) by a nearest neighbor query on the vertex kd-tree. In each iteration, Algorithm 6 finds the neighbor min of the current vertex opt that has the smallest sum of distances among all the neighbors (Line 6). If the neighbor min has a smaller sum of distances than the current vertex opt, we update the current vertex to be $\min$ (Line 9). Otherwise, Algorithm 6 terminates and the current point is returned (Line 8).

As we will see in Section 4, although Algorithm 6 may get stuck in a local optimal point (i.e. all its neighbors have larger sums of distances), its sum-of-distances value is very close to the minimum value. More importantly, Algorithm 6 is often able to find the exact OMP and runs orders of magnitude faster than the algorithms described in Sections 3.1 and 3.2. Thus, the algorithm is extremely suitable for large-scale query processing in real time, and the upper bound estimation of sum-of-distances for accelerating location constraint evaluation in applications such as those mentioned in [19].

\section{EXPERIMENTS}

In this section, we evaluate the performance of our algorithms for the OMP queries by using the road network datasets of 46 states in US from [21], and the datasets of "city of Oldenburg" (OL) and "California" (CA) from [22]. Table 2 in the Appendix describes 31 of the datasets we use, which contains the number of nodes and edges in each dataset. The datasets of the remaining states in US from [21] are not used either because their sizes are too small, or because the datasets are composed of many small connected components. We require that all the vertices belong to the same component since we randomly generate OMP query points on the road networks. Appendix F details the experimental platform configuration and the preprocessing of the datasets.

For each dataset, we generate queries by imposing a rectangular window on the dataset, and all the query points are randomly generated on the part of the road network in the window. Let $W$ denote the distance between the $x$-coordinates of the leftmost vertex and the rightmost vertex on the road network, and $H$ denote the distance between the $y$-coordinates of the highest vertex and the lowest vertex on the road network. We parameterize the size of a window by the parameter $\alpha(<1)$ so that the window has size $\alpha W \times \alpha H$. Appendix $\mathrm{G}$ presents the details of our query generator.

The configuration of a query set $Q$ can be represented as a triple $\left(\alpha, N_{p}, N_{w}\right)$, where $N_{w}$ denotes the number of windows used to generate the query set, $N_{p}$ denotes the number of query points generated in each window, and $\alpha$ decides the size of each window (which is $\alpha W \times \alpha H$ ). The total number of query points is
$|Q|=N_{p} \times N_{w}$. We introduce the parameter $N_{w}$ to generate query sets whose query points belong to several groups, and the points in each group are close to each other. For each dataset and each query set configuration, we randomly generate 100 query sets for evaluation.

We implemented the split point checking algorithm proposed in [19], denoted as Split (SP), for experimental comparison. For fairness of comparison, we use our pre-computed shortest path index for the shortest path computations required by Split.

Besides Split (SP), in the sequel, we denote our baseline algorithm as Baseline (BL), the algorithm that uses only the first phase of convex-hull-based pruning as HullWindow (HW), the one that uses two-phase convex-hull-based pruning as HullWindow2 (HW2), and the greedy algorithm as Greedy (GD).

To fully utilize the pruning in Lines 4-5 of Algorithm 2, we can use the result of Greedy to initialize the current minimum sum-ofdistances value of the other algorithms, i.e. Line 3 of Algorithm 1 and Line 5 of Algorithm 5. We denote the algorithm versions that use Greedy for initialization by appending an apostrophe to the original algorithm names, e.g. Baseline becomes Baseline' (BL').

We evaluate the performance of our algorithms based on the following four criteria: (1) query processing time; (2) the sum-ofdistances ratio of the other algorithms to Baseline; (3) the number of steps (i.e. iterations) of Greedy; and (4) the number of times that each algorithm finds the exact OMP among the 100 queries (for each configuration on each dataset). The reported value of the first three criteria are averaged over the 100 queries.

Due to the space limitation, we only show our results on the "CA" and "OL" datasets from [22], and the overall results of the 47 datasets in this section. Appendix $\mathrm{H}$ shows part of the results on all the datasets, and the complete results are available online ${ }^{12}$.

\subsection{Effect of the Window Size of Query Sets}

Figure 6(a) (Figure 7(a)) shows the average execution time of our algorithms (over 100 queries) for different window sizes (determined by $\alpha$ ), where we set $N_{w}=1$ and $N_{p}=20$ for each query. We can see that Split takes the most time, Baseline the second most, HullWindow2 the third, HullWindow the fourth, and finally Greedy. In all our experiments, Split is found to be stably around an order of magnitude slower than our most expensive algorithm Baseline, which is better than our expectation due to its pruning rule. Besides, initialization using Greedy does improve the performance of the algorithms, and the improvement on OL is more obvious than that on CA. While the query processing time increases with the increment of window size for the other algorithms, Greedy shows rather stable performance for all values of $\alpha$, and usually takes tens of milliseconds. On the average of the 47 datasets in this set of experiments, HullWindow2 runs 2.14 times faster than Baseline, and Greedy runs 142.02 times faster than HullWindow2.

Let $s d(u, Q)$ denote the sum-of-distances value of vertex $u$ for query set $Q$. As Baseline guarantees to return the OMP opt, for some other algorithm that returns the meeting point $v$, we define its sum-of-distances ratio to be $s d(v, Q) / s d($ opt,$Q)-1$. Figure 6(b) (Figure 7(b)) shows the average sum-of-distances ratio of our algorithms. We can see that HullWindow2 always returns the OMP, while HullWindow may return a near-optimal meeting point when $\alpha<40 \%$. The result of Greedy is less optimal but the sum-ofdistances ratio is always within $3.5 \%$.

Figure 6(c) (Figure 7(c)) shows the average number of iterations run by Greedy for different window sizes, and Figure 6(d) (Fig-

\footnotetext{
${ }^{1}$ http://www.cse.ust.hk/ yanda/datasets/part1.pdf

${ }^{2} \mathrm{http}: / /$ www.cse.ust.hk/ yanda/datasets/part2.pdf
} 


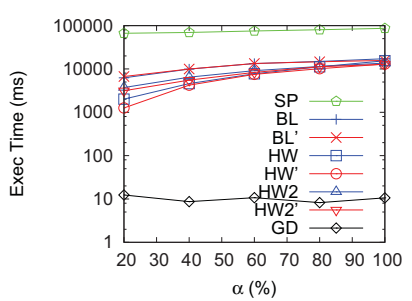

(a) Exec. Time

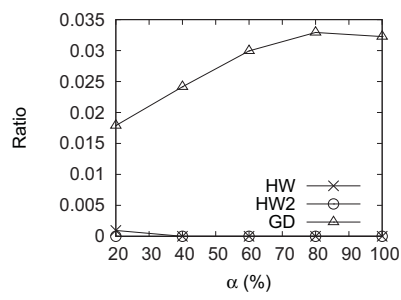

(b) Sum-of-Distances Ratio

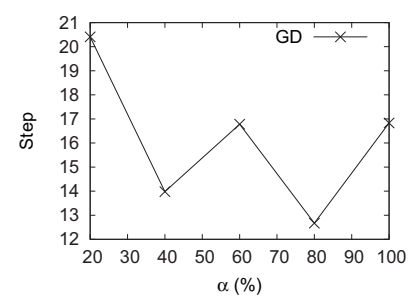

(c) Number of Steps of Greedy

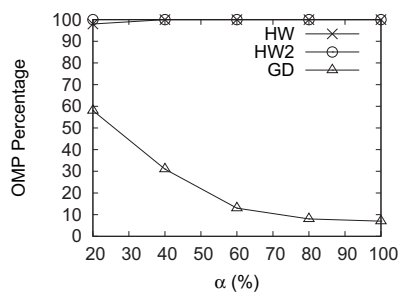

(d) OMP percentage

Figure 6: Effect of the Window Size of Query Sets on the CA Dataset.

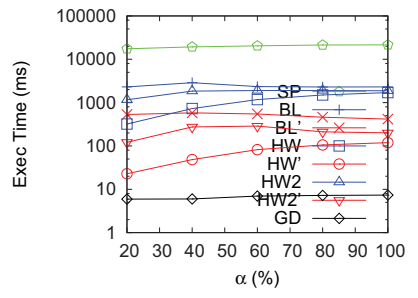

(a) Exec. Time

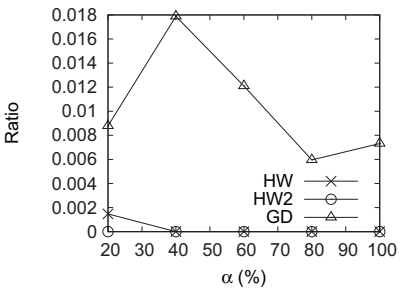

(b) Sum-of-Distances Ratio

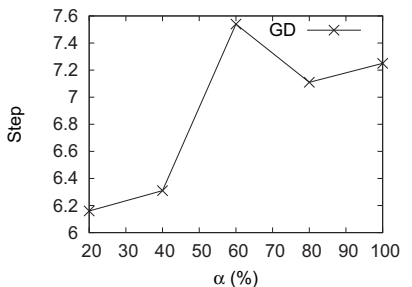

(c) Number of Steps of Greedy

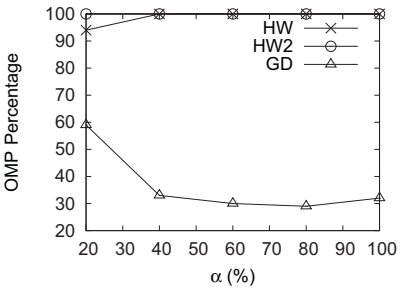

(d) OMP percentage

Figure 7: Effect of the Window Size of Query Sets on the OL Dataset.

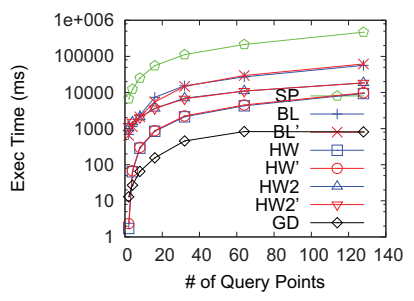

(a) Exec. Time

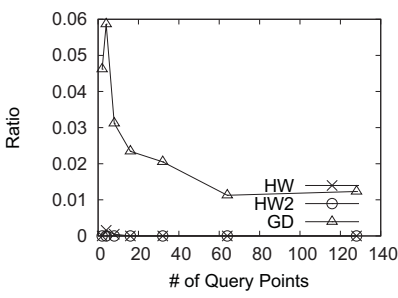

(b) Sum-of-Distances Ratio

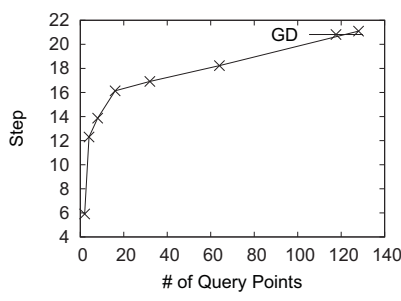

(c) Number of Steps of Greedy

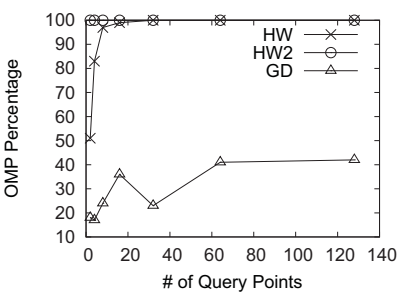

(d) OMP percentage

Figure 8: Effect of the Number of Query Points on the CA Dataset.

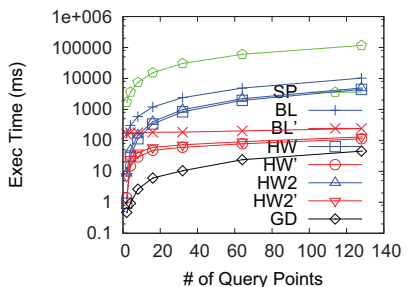

(a) Exec. Time

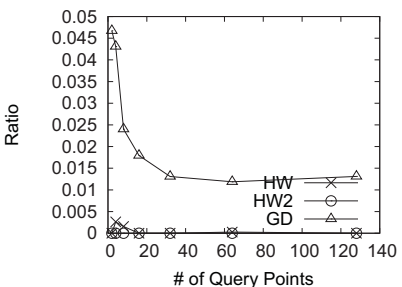

(b) Sum-of-Distances Ratio

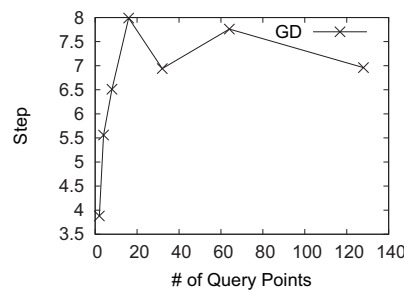

(c) Number of Steps of Greedy

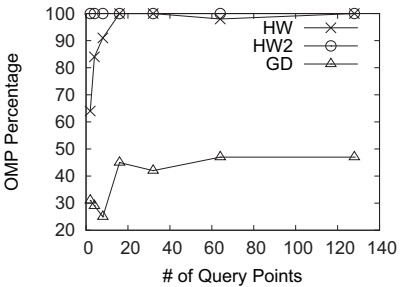

(d) OMP percentage

Figure 9: Effect of the Number of Query Points on the OL Dataset.

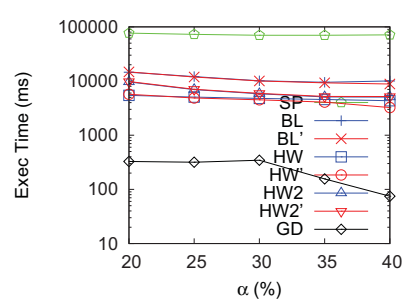

(a) Exec. Time

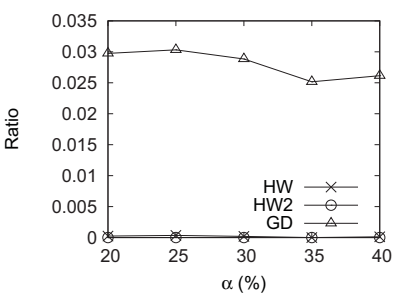

(b) Sum-of-Distances Ratio

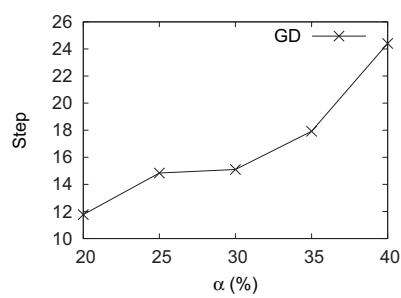

(c) Number of Steps of Greedy

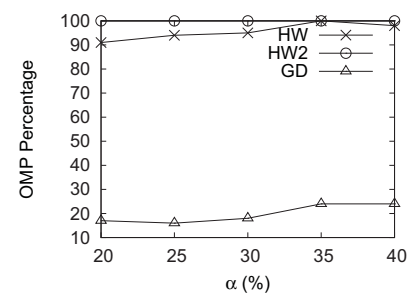

(d) OMP percentage

Figure 10: Effect of Multiple Windows on the CA Dataset. 
ure 7(d)) shows the number of times that the algorithms return the exact OMP among the 100 queries. HullWindow 2 always returns the OMP, while HullWindow returns the OMP for over $90 \%$ of the queries, the percentage of which reaches $100 \%$ as $\alpha$ increases to $40 \%$. On the other hand, Greedy manages to return the OMP for only a small fraction of the queries, and the percentage goes down with the increment of $\alpha$.

\subsection{Effect of the Number of Query Points}

Figures 8 (Figures 9) (a)-(d) show the results of our algorithms for different query set sizes $\left(N_{p}=2^{i}, i \in\{1,2, \ldots, 7\}\right)$. In this set of experiments, we set $N_{w}=1$ and $\alpha=40 \%$. From Figures 8 (Figures 9) (a) and (d), we can obtain similar observations as in Section 4.1. We find that HullWindow2 can be tens of times faster than Baseline for small $N_{p}$, but the gap reduces to only several times when $N_{p}$ becomes larger. On the average of the 47 datasets in this set of experiments, HullWindow2 runs 5.88 times faster than Baseline, and Greedy runs 54 times faster than HullWindow2.

We can see from Figure 8(b) (Figure 9(b)) that the average sumof-distances ratio of Greedy gets smaller as there are more query points in the query set, which stabilizes at between $1 \%$ and $1.5 \%$ when $|Q| \geq 60$. Figure 8(c) (Figure 9(c)) shows that Greedy requires more iterations when $|Q|$ becomes larger.

\subsection{Effect of Multiple Windows}

In this set of experiments, we study the scenario where the query points belong to several groups, and the points in each group are close to each other. Specifically, we study the case where there are two groups and each group contains 10 query points. We set $N_{w}=$ $2, N_{p}=10$, and vary the parameter $\alpha$ to see the performance of our algorithms for different window sizes.

Figures 10 (Figures 11 in the Appendix) (a)-(d) show the results of our algorithms for different query set sizes (determined by $N_{p}$ ). We find that the performance of all the algorithms are quite stable: As $\alpha$ increases, the query processing time does not increase, the sum-of-distances ratio decreases, and the number of iterations of Greedy increases. On the average of the 47 datasets in this set of experiments, HullWindow2 runs 2.51 times faster than Baseline, and Greedy runs 129.9 times faster than HullWindow2.

Among the OMP 79900 queries in total, HullWindow2 only fails to return the OMP for 5 queries, and the sum-of-distances ratio of these result meeting points are all within $0.1 \%$. Therefore, HullWindow2 is a good alternative to Baseline, especially for small query sets. Greedy is over two orders of magnitude faster than the other algorithms, and usually takes only tens of milliseconds. Besides, its sum-of-distances ratio is usually around 3\%. Therefore, the meeting point returned by Greedy is of high quality, and Greedy is the most practical method to support large-scale meeting point queries on real-world spatial database servers.

\section{CONCLUSION}

In this paper, we study the optimal meeting point query that returns the point on a road network $G=(V, E)$ with the smallest sum of network distances to all the query points in a given query set $Q$. Our baseline algorithm substantially reduces the search space of the OMP query from $|Q| \cdot|E|$ to $|V|+|Q|$ according to the spatial property established in Theorem 1 . We also design an effective two-phase convex-hull-based pruning technique to further prune the search space. Finally, we develop an extremely efficient greedy algorithm to find a high-quality near-optimal meeting point instead of an exact OMP. The efficiency of this algorithm makes it the most practical choice for spatial applications that involve large flow of queries and require fast response as the top priority.
ACKNOWLEDGEMENTS: We are grateful to the anonymous reviewers for their insightful comments on this paper. This work is partially supported by RGC GRF under grant number HKUST 618509.

\section{REFERENCES}

[1] N. Beckmann, H. Kriegel, R. Schneider and B. Seeger. "The $\mathrm{R}^{*}$-Tree: An efficient and robust access method for points and rectangles". In SIGMOD, pp. 322-331, 1990.

[2] S. Boyd and L. Vandenberghe. "Convex Optimization". Cambridge University Press. http://www.stanford.edu/ boyd/cvxbook/bv_cvxbook.pdf

[3] R. Chen. "Location Problems with Costs Being Sums of Powers of Euclidean Distances". Computers \& Mathematics with Applications, vol. 10, issue 1, pp. 87-94, Dec. 1984.

[4] R. Chen. "Solution of Location Problems with Radial Cost Functions". Computers \& Mathematics with Applications, vol. 10, issue 1, pp. 87-94, Dec. 1984.

[5] Z. Chen, H. T. Shen, X. Zhou and J. X. Yu. "Monitoring Path Nearest Neighbor in Road Networks". In SIGMOD, pp. 591-602, 2009.

[6] H. Cho and C. Chung. "An Efficient and Scalable Approach to CNN Queries in a Road Network". In $V L D B$, pp. 865-876, 2005.

[7] L. Cooper. "An Extension of the Generalized Weber Problem". Journal of Regional Science, vol. 8, issue 2, pp. 181-197, Dec. 1968.

[8] L. Cooper. "The Mulitfacility Location Problem: Applications and Descent Theorems". Journal of Regional Science, vol. 17, issue 3, pp. 409-419, Dec. 1977.

[9] G. Hjaltason and H. Samet. "Distance Browsing in Spatial Databases". In TODS, pp. 265-318, 1999.

[10] H. Hu, D. L. Lee and V. C. S. Lee. "Distance Indexing on Road Networks". In VLDB, pp. 894-905, 2006.

[11] M. R. Kolahdouzan and C. Shahabi. "Voronoi-Based $k$ Nearest Neighbor Search for Spatial Network Databases". In $V L D B$, pp. 840-851, 2004.

[12] K. Mouratidis, M. L. Yiu, D. Papadias and N. Mamoulis. "Continuous Nearest Neighbor Monitoring in Road Networks". In VLDB, pp. 43-54, 2006.

[13] D. Papadias, Q. Shen, Y. Tao and K. Mouratidis. "Group Nearest Neighbor Queries”. In ICDE, pp. 301-312, 2004.

[14] D. Papadias, J. Zhang, N. Mamoulis and Y. Tao. "Query Processing in Spatial Network Databases". In $V L D B$, pp. 802-813, 2003.

[15] F. P. Preparata and M. I. Shamos. "Computational Geometry: An Introduction”. Springer-Verlag, 1985.

[16] H. Samet, J. Sankaranarayanan and H. Alborzi. "Scalable Network Distance Browsing in Spatial Databases". In SIGMOD, pp. 43-54, 2008.

[17] Y. Tao, D. Papadias and Q. Shen. "Continuous Nearest Neighbor Search”. In VLDB, pp. 287-298, 2002.

[18] Z. Xu and H.-A. Jacobsen. "Efficient Constraint Processing for Location-aware Computing”. In MDM, pp. 3-12, 2005.

[19] Z. Xu and H.-A. Jacobsen. "Processing Proximity Relations in Road Networks". In SIGMOD, pp. 243-254, 2010.

[20] M. L. Yiu, N. Mamoulis and D. Papadias. "Aggregate Nearest Neighbor Queries in Road Networks". In TKDE, pp. 820-833, 2005.

[21] http://data.geocomm.com/catalog/US

[22] http://www.cs.fsu.edu/ lifeifei/SpatialDataset.htm 


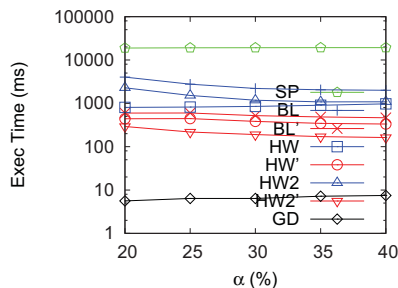

(a) Exec. Time

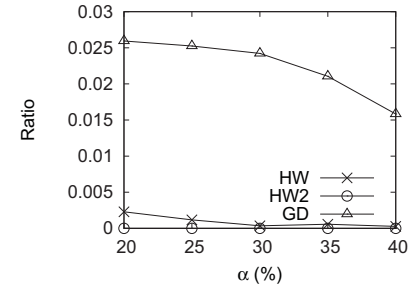

(b) Sum-of-Distances Ratio

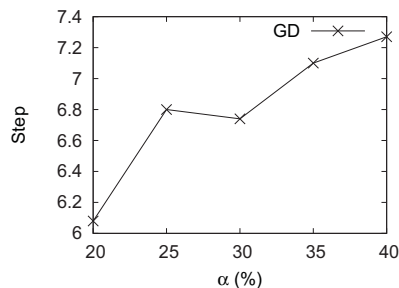

(c) Number of Steps of Greedy

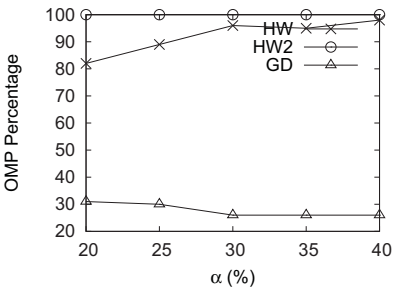

(d) OMP percentage

Figure 11: Effect of Multiple Windows on the OL Dataset.

Table 2: DataSet Description

\begin{tabular}{|c|c|c||c|c|c||c|c|c||c|c|c|}
\hline data & vertex \# & edge \# & data & vertex \# & edge \# & data & vertex \# & edge \# & data & vertex \# & edge \# \\
\hline CA & 21048 & 21693 & FL & 6879 & 7303 & LA & 5886 & 6133 & NY & 7503 & 7766 \\
\hline OL & 6105 & 7035 & GA & 6757 & 7038 & MA & 1530 & 1595 & OH & 4738 & 4986 \\
\hline AR & 7454 & 7695 & IA & 4833 & 5168 & MD & 1222 & 1270 & OK & 6877 & 7300 \\
\hline AZ & 11050 & 11381 & ID & 8108 & 8310 & ME & 4536 & 4656 & PA & 6384 & 6619 \\
\hline CO & 9796 & 10175 & IL & 6117 & 6520 & MI & 5928 & 6198 & RI & 203 & 212 \\
\hline CT & 923 & 958 & IN & 4300 & 4592 & MN & 7718 & 8184 & SC & 2868 & 2966 \\
\hline DE & 250 & 257 & KS & 5615 & 6054 & MO & 9500 & 9889 & SD & 6733 & 7165 \\
\hline DR & 10513 & 10738 & KY & 4745 & 4952 & MS & 6022 & 6297 & & & \\
\hline
\end{tabular}

\section{APPENDIX}

\section{A. PROOF OF THEOREM 2}

Theorem 2: Given a point set $Q=\left\{q_{1}, q_{2}, \ldots, q_{n}\right\}$ on an arbitrary graph $G=(V, E)$, where each point $q_{i}$ is associated with a weight $w_{i}$. If all the weights are integers or rational numbers, then $V \cup Q$ must contain the point $\bar{x}=\arg \min _{x} \sum_{i} w_{i} \cdot d\left(\overline{q_{i}, x}\right)$.

PROOF. It is straightforward to convert the rational number weights into integer weights with the same weight distribution among all the points in $Q$. For example, suppose $Q=\left\{q_{1}, q_{2}, q_{3}\right\}, w_{1}=$ $0.15, w_{2}=1.11$ and $w_{3}=0.8$, then we can re-assign the weights to be $w_{1}=15, w_{2}=111$ and $w_{3}=80$. Clearly, this transformation does not change the result point $\bar{x}$.

Now, let us assume that all the weights are integers, and we replace each point $q_{i}$ with $w_{i}$ new points at the same location of $q_{i}$, each of which has weight 1 . The resulting new query point set $Q^{\prime}$ can be treated as unweighted, and thus $Q^{\prime} \cup V$ contains $\bar{x}$ according to Theorem 1. It is straightforward to see that the transformation from $Q$ to $Q^{\prime}$ does not change the result point $\bar{x}$, and the locations in $Q^{\prime}$ is exactly the locations in $Q$. Therefore, Theorem 2 is proved.

\section{B. DISCUSSION ON THEOREM 1 AND SPLIT-POINT-BASED OMP ALGORITHM}

Theorem 1 states that we can find an OMP among the vertices and query points, while [19] states that we can find an OMP among the split points. Although the two methods seems to be contradictory, they are both correct because (1)OMP may not be unique, and (2)OMP can be both a split point and a vertex.

The first reason is easy to understand: consider a query point set $Q=\left\{q_{1}, q_{2}\right\}$, then any point on the shortest path $\overline{p_{1}, p_{2}}$ can be the OMP, including all the split points and vertices on $\overline{p_{1}, p_{2}}$.

Now let us discuss the second reason. For a point $p$ on a road network, its split point on edge $(u, v)$ is defined by [19] to be the point $s$ such that

$$
d(\overline{p, u})+d(u \sim s)=d(\overline{p, v})+d(v \sim s)
$$

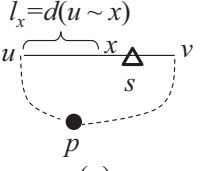

(a)

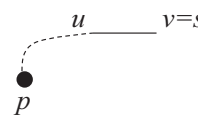

(b)

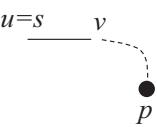

(c)

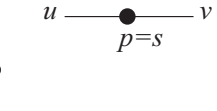

(d)
Figure 12: Four Cases for Split Points.

However, this definition is only suitable for the scenario that $p$ is not on $(u, v)$, which can be further divided into three cases illustrated by Figure 12(a)-(c) where the dotted curves denote the shortest paths:

- Case 1: $\overline{p, v}$ does not pass through $u$, and $\overline{p, u}$ does not pass through $v$ (Figure 12(a)). In this case, the split point $s$ exists due to the fact that the road network is a metric space, i.e. $d(\overline{p, u})+d(u \sim v) \geq d(\overline{p, v})$ and $d(\overline{p, v})+d(u \sim v) \geq$ $d(\overline{p, u})$. If we represent an arbitrary point $x$ on $(u, v)$ using $l_{x}=d(u \sim x)$, we can see that $d(\overline{p, x})$ is a piecewise linear function to $l_{x}$ on $(u, v)$ which consists of two different linear functions defined on $\left[0, l_{s}\right]$ and $\left[l_{s}, l_{v}\right]$.

- Case 2: $\overline{p, v}$ passes through $u$ (Figure 12(b)). In this case, the split point is vertex $v$, as can be checked using Equation (1). For an arbitrary point $x$ on $(u, v), d(\overline{p, x})=d(\overline{p, u})+$ $l_{x}$ is a function linear to $l_{x}$.

- Case 3: $\overline{p, u}$ passes through $v$ (Figure 12(c)). In this case, the split point is vertex $u$, as can be checked using Equation (1). For an arbitrary point $x$ on $(u, v), d(\overline{p, x})=d(\overline{p, u})-$ $l_{x}$ is a function linear to $l_{x}$.

When $p$ is on $(u, v)$ (Figure 12(d)), we define $p$ to be the split point $s$ so that $d(\overline{p, x})=\left|l_{s}-l_{x}\right|$ is still a piecewise linear function delimited by $s$.

Therefore, for each query point $p$ and a point $x$ on $(u, v), d(\overline{p, x})$ is a piecewise linear function delimited by the split point $s$. Since the sum-of-distances value $\sum_{p \in Q} d(\overline{p, x})$ is the sum of piecewise linear functions delimited by the split points, it achieves the mini- 
mum or maximum at the split points on $(u, v)$. This establishes the correctness of the split point checking method in [19].

Note that for the cases illustrated by Figures 12(b)-(d), the split points belong to $V \cup Q$ which are those checked by Theorem 1 . In fact, our experiments show that the vast majority of the OMPs returned by the split point checking method in [19] belong to Cases 2 and 3. Although both methods are proved to be correct, our method checks $|V|+|Q|$ candidate points, which is much smaller than the $|Q| \cdot|E|$ split points computed and checked by [19].

\section{SHORTEST PATH INDEX BETWEEN VERTICES}

Since the definition of OMP on a road network is directly built on shortest paths, we construct a disk-based index file to accelerate the shortest path computation between two vertices on the road network. Shortest path computation is actually the basic operation of many queries on the road network $[19,16,10,14,5]$. While a lot of efficient online algorithms have been proposed $[14,5]$ for shortest path computation on road networks, [16] presents a nice off-line index for shortest path computation on road networks. As road networks seldomly change, off-line indices are usually viable and more effective in shortening query execution time.

As shortest path computation is not our main focus, we simply run Dijkstra algorithm for each vertex in the road network, to compute $d\left(\overline{v_{1}, v_{2}}\right)$ for each pair of vertices $v_{1}$ and $v_{2}$. The result is a 2D array, which is written to a file. During query processing, to obtain $d\left(\overline{v_{1}, v_{2}}\right)$, we set the file pointer to the location where it is stored and read the value. Therefore, only one I/O operation is required to obtain $d\left(\overline{v_{1}, v_{2}}\right)$.

To find the shortest path between two vertices, we maintain another disk-based index which is also built by the Dijkstra algorithm, together with the shortest path length index mentioned above. Besides the length of the shortest path from vertex $v_{1}$ to $v_{2}$, the Dijkstra algorithm also reports the vertex before $v_{2}$ on the shortest path from $v_{1}$ to $v_{2}$, which can be used to find the shortest path by going back iteratively. We store another 2D array Path on the disk, which is appended at the end of shortest path length index in the index file, where Path $\left[v_{1}\right]\left[v_{2}\right]$ stores the first vertex after $v_{1}$ that is on the shortest path from $v_{1}$ to $v_{2}$. Algorithm 7 shows the way to find the shortest path $\mathcal{L}$ between $v_{1}$ and $v_{2}$, which takes $\ell \mathrm{I} / \mathrm{O}$ operations, where $\ell$ denotes the length of $\mathcal{L}$.

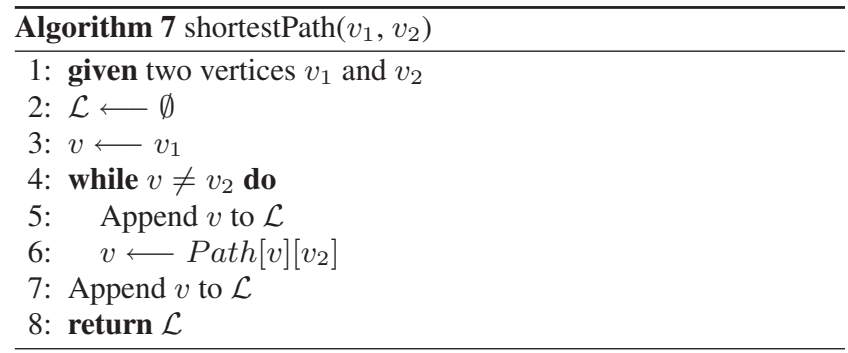

Note that the more sophisticated methods for shortest path computation mentioned in the beginning of Appendix $\mathrm{C}$ can also be used, since our algorithms for the OMP query do not have specific requirement on the shortest path computation.

\section{SHORTEST PATH COMPUTATION BE- TWEEN ARBITRARY POINTS}

The length of the shortest path from a query point $q$ on edge $(u, v)$ to a vertex $p$ is computed as $d(\overline{q, p})=\min \{d(q \sim u)+$ $d(\overline{u, p}), d(q \sim v)+d(\overline{v, p})\}$, which requires two I/O operations from the index.

The length of the shortest path from a query point $q$ on edge $(u, v)$ to another query point $q^{\prime}$ on edge $\left(u^{\prime}, v^{\prime}\right)$ is computed as $d\left(\overline{q, q^{\prime}}\right)=\min \left\{d(q \sim u)+d\left(\overline{u, u^{\prime}}\right)+d\left(u^{\prime} \sim q^{\prime}\right), d(q \sim u)+\right.$ $d\left(\overline{u, v^{\prime}}\right)+d\left(v^{\prime} \sim q^{\prime}\right), d(q \sim v)+d\left(\overline{v, u^{\prime}}\right)+d\left(u^{\prime} \sim q^{\prime}\right), d(q \sim$ $\left.v)+d\left(\overline{v, v^{\prime}}\right)+d\left(v^{\prime} \sim q^{\prime}\right)\right\}$, if $q$ and $q^{\prime}$ are on different edges, which requires four $\mathrm{I} / \mathrm{O}$ operations from the index.

If $q$ and $q^{\prime}$ are on the same edge, then according to the triangular inequality of the road network edges, the shortest path length $d\left(\overline{q, q^{\prime}}\right)=d\left(q \sim q^{\prime}\right)$, which requires no I/O operation.

\section{E. COUNTEREXAMPLES}

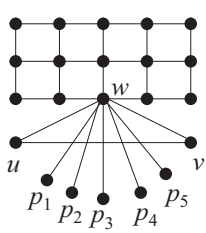

(a)

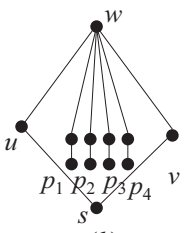

(b)
Figure 13: Counterexamples.

Figure 13(a) shows an example where the road network partitioning scheme of [19] fails to obtain the OMP. Let us assume that a query point is at each vertex in the set $S=\left\{u, p_{1}, p_{2}, p_{3}, p_{4}, p_{5}, v\right\}$, and that the edge $(u, v)$ cuts the graph into two partitions. Since all the query points belong to one partition (below $(u, v)$ ), the partitioning scheme of [19] will not check the vertex $w$ (above $(u, v)$ ). However, by simple reasoning, we can see that $w$ is the OMP.

Our two-phase online convex-hull-based pruning technique is able to find the OMP for the above example. This is because $p_{1}$ and $p_{2}$ are two neighboring points on the convex hull of the point set $S$, and the shortest path between them is $\left(p_{1}, w, p_{2}\right)$. As a result, $w$ will be included in the second phase for further checking.

However, our technique may also have some limitation in some extreme cases. Consider the example road network in Figure 13(b), where we assume that a query point is at each vertex in the set $S^{\prime}=\left\{u, p_{1}, p_{2}, p_{3}, p_{4}, v, s\right\}$. In the first phase pruning, we obtain the convex hull of $S^{\prime}$, which contains $u, s$ and $v$. In the second phase pruning, $w$ will then not be included as none of the three shortest paths between the points in $\{u, v, s\}$ goes through $w$. As a result, $w$ is not checked by our technique, although it is easy to check that $w$ is the OMP.

We assume in the above example that the query point at $u$ is represented as on an edge other than $(u, w)$ (See Definition 1), e.g. $(u, s)$, since otherwise, $w$ will be included in the first phase. There is a similar assumption for $v$. Note that the intermediate point(s) between $p_{i}$ and $w$ is also important for the construction of the counterexample, since otherwise, $p_{i}$ is represented as on edge $\left(p_{i}, w\right)$ and $w$ will be included. As real road networks are usually of regular structure rather than the weird topology as given in Figure 13(b), we claim in this sense that our two-phase online convex-hull-based pruning technique is able to find the OMP on almost all real road networks.

\section{F. EXPERIMENTAL SETTING AND DATASET PREPROCESSING}

All the experiments are done on a computer with Intel(R) Core(TM) i5 CPU and 4GB memory. All our programs are written in JAVA, and run in Eclipse on Windows 7 Enterprise. 
We use the road network datasets of 46 states in US from [21], and the datasets of "city of Oldenburg" (OL) and "California" (CA) from [22] for experiments. As the "CA" dataset is contained in both sources, we only use the one from [22] for the experiment. Since we randomly generate OMP query points on the road networks, if two query points are in two different connected components, they can never reach each other and the OMP does not exist. Therefore, we require that all the vertices belong to the same component, and do not use the datasets of the remaining states in US from [21] that are composed of many small connected components.

While the datasets from [22] are directly usable, the datasets from [21] are in E00 file format. We use the "Global Mapper" software $^{3}$ to convert the datasets into readable raw data files. After merging the duplicate vertices, we get the largest connected components of each dataset (which usually contains over $99 \%$ of the original vertices). The preprocessed datasets are available from

http://www. cse.ust.hk/ yanda/datasets/roadData.rar

\section{G. QUERY GENERATOR}

To generate a query point in a window, we find all the edges of the road network that intersect with the window, and randomly pick an edge from them. After picking the edge, we randomly generate a query point on the segment of the edge that is in the window.

To generate a query set for window parameter $\alpha$, we randomly position a $\alpha W \times \alpha H$ rectangular window in the whole 2D space for the road network. If there are less than $\epsilon$ edges covered by the window, we drop it and generate a new window. This process is repeated until a window with at least $\epsilon$ edges is generated, and then we generate $|Q|$ query points using the method described in the previous paragraph. The parameter $\epsilon$ is used to avoid generating a window in a sparse area of the space of the road network, and is set to 20 throughout our experiments.

\section{H. ADDITIONAL EXPERIMENT RESULTS}

Due to the space limitation, in Section 4, we only show our experimental results on two of the 47 datasets, i.e. CA and OL. As the complete results still contain too many entries to be put in the appendix, we make them available online:

- The running time of our various algorithms and the number of steps executed by Greedy under different query configurations on all the datasets are recorded at:

http://www. cse.ust.hk/ Yyanda/datasets/part1.pdf

- The sum-of-distance ratio of our various algorithms and the percentage of queries for which each algorithm returns the exact OMP (OMP percentage) under different query configurations on all the datasets are recorded at:

http://www. cse. ust.hk/ Yyanda/datasets/part2.pdf

In the sequel, we show part of our results on sum-of-distance ratio and $O M P$ percentage for 31 of the datasets in all the 3 set of experiments we conduct. In the following tables, $r($.$) in the table$ heads denotes the sum-of-distance ratio of the algorithm, and $o($. denotes the OMP percentage of the algorithm.

- Table 3 shows our results for the experimental setting in Section 4.1 when $\alpha=20 \%$ and $\alpha=100 \%$.

- Table 4 shows our results for the experimental setting in Section 4.2 when $N_{p}=2$ and $N_{p}=128$

- Table 5 shows our results for the experimental setting in Section 4.3 when $\alpha=20 \%$ and $\alpha=40 \%$

\footnotetext{
${ }^{3}$ http://www.globalmapper.com
}

Table 3: Effect of the Window Size of Query Sets.

\begin{tabular}{|c|c|c|c|c|c|c|c|}
\hline data & $\alpha$ & $r(H W)$ & $r(H W 2)$ & $r(G D)$ & $o(H W)$ & $o(H W 2$ & $o(G D)$ \\
\hline $\mathrm{CA}$ & $20 \%$ & $0.094 \%$ & $0 \%$ & $1.788 \%$ & $98 \%$ & $100 \%$ & $58 \%$ \\
\hline CA & $100 \%$ & $0 \%$ & $0 \%$ & $3.225 \%$ & $100 \%$ & $100 \%$ & $7 \%$ \\
\hline $\mathrm{OL}$ & $20 \%$ & $0.147 \%$ & $0 \%$ & $0.878 \%$ & $94 \%$ & $100 \%$ & $59 \%$ \\
\hline $\mathrm{OL}$ & $100 \%$ & $0 \%$ & $0 \%$ & $0.732 \%$ & $100 \%$ & $100 \%$ & $32 \%$ \\
\hline AR & $20 \%$ & $0 \%$ & $0 \%$ & $1.374 \%$ & $100 \%$ & $100 \%$ & $66 \%$ \\
\hline AR & $100 \%$ & $0 \%$ & $0 \%$ & $2.156 \%$ & $100 \%$ & $100 \%$ & $22 \%$ \\
\hline AZ & $20 \%$ & $0.018 \%$ & $0 \%$ & $1.525 \%$ & $96 \%$ & $100 \%$ & $69 \%$ \\
\hline AZ & $100 \%$ & $0 \%$ & $0 \%$ & $2.124 \%$ & $100 \%$ & $100 \%$ & $22 \%$ \\
\hline $\mathrm{CO}$ & $20 \%$ & $0 \%$ & $0.067 \%$ & $1.558 \%$ & $100 \%$ & $99 \%$ & $55 \%$ \\
\hline $\mathrm{CO}$ & $100 \%$ & $0 \%$ & $0 \%$ & $2.082 \%$ & $100 \%$ & $100 \%$ & $20 \%$ \\
\hline $\mathrm{CT}$ & $20 \%$ & $0.02 \%$ & $0 \%$ & $0.358 \%$ & $96 \%$ & $100 \%$ & $69 \%$ \\
\hline CT & $100 \%$ & $0.004 \%$ & $0 \%$ & $1.459 \%$ & $99 \%$ & $100 \%$ & $74 \%$ \\
\hline $\mathrm{DE}$ & $20 \%$ & $0.021 \%$ & $0 \%$ & $0.601 \%$ & $99 \%$ & $100 \%$ & $66 \%$ \\
\hline DE & $100 \%$ & $0 \%$ & $0 \%$ & $0.084 \%$ & $100 \%$ & $100 \%$ & $85 \%$ \\
\hline DR & $20 \%$ & $0.03 \%$ & $0 \%$ & $1.192 \%$ & $98 \%$ & $100 \%$ & $64 \%$ \\
\hline DR & $100 \%$ & $0 \%$ & $0 \%$ & $1.649 \%$ & $100 \%$ & $100 \%$ & $36 \%$ \\
\hline FL & $20 \%$ & $0.103 \%$ & $0 \%$ & $1.439 \%$ & $95 \%$ & $100 \%$ & $57 \%$ \\
\hline FL & $100 \%$ & $0 \%$ & $0 \%$ & $3.598 \%$ & $100 \%$ & $100 \%$ & $3 \%$ \\
\hline GA & $20 \%$ & $0.032 \%$ & $0 \%$ & $1.985 \%$ & $97 \%$ & $100 \%$ & $67 \%$ \\
\hline GA & $100 \%$ & $0 \%$ & $0 \%$ & $1.352 \%$ & $100 \%$ & $100 \%$ & $45 \%$ \\
\hline IA & $20 \%$ & $0.039 \%$ & $0 \%$ & $1.554 \%$ & $98 \%$ & $100 \%$ & $61 \%$ \\
\hline IA & $100 \%$ & $0 \%$ & $0 \%$ & $1.306 \%$ & $100 \%$ & $100 \%$ & $24 \%$ \\
\hline ID & $20 \%$ & $0.179 \%$ & $0 \%$ & $1.021 \%$ & $90 \%$ & $100 \%$ & $58 \%$ \\
\hline ID & $100 \%$ & $0.002 \%$ & $0 \%$ & $3.571 \%$ & $99 \%$ & $100 \%$ & $14 \%$ \\
\hline IL & $20 \%$ & $0.002 \%$ & $0 \%$ & $1.59 \%$ & $99 \%$ & $100 \%$ & $65 \%$ \\
\hline IL & $100 \%$ & $0 \%$ & $0 \%$ & $1.191 \%$ & $100 \%$ & $100 \%$ & $19 \%$ \\
\hline IN & $20 \%$ & $0 \%$ & $0 \%$ & $1.78 \%$ & $100 \%$ & $100 \%$ & $63 \%$ \\
\hline IN & $100 \%$ & $0 \%$ & $0 \%$ & $2.254 \%$ & $100 \%$ & $100 \%$ & $17 \%$ \\
\hline KS & $20 \%$ & $0.069 \%$ & $0 \%$ & $1.597 \%$ & $97 \%$ & $100 \%$ & $61 \%$ \\
\hline KS & $100 \%$ & $0 \%$ & $0 \%$ & $1.318 \%$ & $100 \%$ & $100 \%$ & $18 \%$ \\
\hline KY & $20 \%$ & $0.072 \%$ & $0 \%$ & $1.215 \%$ & $95 \%$ & $100 \%$ & $59 \%$ \\
\hline KY & $100 \%$ & $0 \%$ & $0 \%$ & $2.6 \%$ & $100 \%$ & $100 \%$ & $15 \%$ \\
\hline LA & $20 \%$ & $0.074 \%$ & $0 \%$ & $1.189 \%$ & $97 \%$ & $100 \%$ & $65 \%$ \\
\hline LA & $100 \%$ & $0 \%$ & $0 \%$ & $1.807 \%$ & $100 \%$ & $100 \%$ & $57 \%$ \\
\hline MA & $20 \%$ & $0.106 \%$ & $0 \%$ & $0.425 \%$ & $94 \%$ & $100 \%$ & $75 \%$ \\
\hline MA & $100 \%$ & $0 \%$ & $0 \%$ & $1.498 \%$ & $100 \%$ & $100 \%$ & $44 \%$ \\
\hline MD & $20 \%$ & $0.016 \%$ & $0 \%$ & $0.606 \%$ & $95 \%$ & $100 \%$ & $58 \%$ \\
\hline MD & $100 \%$ & $0 \%$ & $0 \%$ & $1.645 \%$ & $100 \%$ & $100 \%$ & $39 \%$ \\
\hline ME & $20 \%$ & $0.109 \%$ & $0 \%$ & $1.144 \%$ & $97 \%$ & $100 \%$ & $72 \%$ \\
\hline ME & $100 \%$ & $0 \%$ & $0 \%$ & $2.758 \%$ & $100 \%$ & $100 \%$ & $25 \%$ \\
\hline MI & $20 \%$ & $0.144 \%$ & $0 \%$ & $1.254 \%$ & $97 \%$ & $100 \%$ & $59 \%$ \\
\hline MI & $100 \%$ & $0 \%$ & $0 \%$ & $4.746 \%$ & $100 \%$ & $100 \%$ & $10 \%$ \\
\hline MN & $20 \%$ & $0.031 \%$ & $0 \%$ & $0.834 \%$ & $99 \%$ & $100 \%$ & $64 \%$ \\
\hline MN & $100 \%$ & $0 \%$ & $0 \%$ & $1.659 \%$ & $100 \%$ & $100 \%$ & $17 \%$ \\
\hline MO & $20 \%$ & $0.001 \%$ & $0 \%$ & $1.483 \%$ & $99 \%$ & $100 \%$ & $60 \%$ \\
\hline MO & $100 \%$ & $0 \%$ & $0 \%$ & $1.999 \%$ & $100 \%$ & $100 \%$ & $9 \%$ \\
\hline MS & $20 \%$ & $0.058 \%$ & $0 \%$ & $1.013 \%$ & $97 \%$ & $100 \%$ & $74 \%$ \\
\hline MS & $100 \%$ & $0 \%$ & $0 \%$ & $2.452 \%$ & $100 \%$ & $100 \%$ & $24 \%$ \\
\hline MT & $20 \%$ & $0.478 \%$ & $0 \%$ & $1.815 \%$ & $89 \%$ & $100 \%$ & $53 \%$ \\
\hline MT & $100 \%$ & $0 \%$ & $0 \%$ & $1.83 \%$ & $100 \%$ & $100 \%$ & $30 \%$ \\
\hline $\mathrm{NC}$ & $20 \%$ & $0.177 \%$ & $0 \%$ & $1.311 \%$ & $93 \%$ & $100 \%$ & $59 \%$ \\
\hline $\mathrm{NC}$ & $100 \%$ & $0 \%$ & $0 \%$ & $1.744 \%$ & $100 \%$ & $100 \%$ & $27 \%$ \\
\hline ND & $20 \%$ & $0 \%$ & $0 \%$ & $1.149 \%$ & $100 \%$ & $100 \%$ & $56 \%$ \\
\hline ND & $100 \%$ & $0 \%$ & $0 \%$ & $1.548 \%$ & $100 \%$ & $100 \%$ & $19 \%$ \\
\hline NE & $20 \%$ & $0.078 \%$ & $0 \%$ & $1.342 \%$ & $96 \%$ & $100 \%$ & $60 \%$ \\
\hline $\mathrm{NE}$ & $100 \%$ & $0 \%$ & $0 \%$ & $1.713 \%$ & $100 \%$ & $100 \%$ & $19 \%$ \\
\hline $\mathrm{NH}$ & $20 \%$ & $0.007 \%$ & $0 \%$ & $0.546 \%$ & $97 \%$ & $100 \%$ & $73 \%$ \\
\hline $\mathrm{NH}$ & $100 \%$ & $0 \%$ & $0 \%$ & $1.929 \%$ & $100 \%$ & $100 \%$ & $41 \%$ \\
\hline NJ & $20 \%$ & $0.093 \%$ & $0 \%$ & $0.187 \%$ & $97 \%$ & $100 \%$ & $76 \%$ \\
\hline NJ & $100 \%$ & $0 \%$ & $0 \%$ & $2.381 \%$ & $100 \%$ & $100 \%$ & $26 \%$ \\
\hline NM & $20 \%$ & $0.003 \%$ & $0 \%$ & $0.862 \%$ & $99 \%$ & $100 \%$ & $70 \%$ \\
\hline NM & $100 \%$ & $0 \%$ & $0 \%$ & $2.053 \%$ & $100 \%$ & $100 \%$ & $19 \%$ \\
\hline NV & $20 \%$ & $0 \%$ & $0 \%$ & $1.846 \%$ & $100 \%$ & $100 \%$ & $63 \%$ \\
\hline NV & $100 \%$ & $0 \%$ & $0 \%$ & $3.179 \%$ & $100 \%$ & $100 \%$ & $32 \%$ \\
\hline NY & $20 \%$ & $0.257 \%$ & $0 \%$ & $1.227 \%$ & $93 \%$ & $100 \%$ & $61 \%$ \\
\hline NY & $100 \%$ & $0 \%$ & $0 \%$ & $2.348 \%$ & $100 \%$ & $100 \%$ & $17 \%$ \\
\hline $\mathrm{OH}$ & $20 \%$ & $0.04 \%$ & $0 \%$ & $1.281 \%$ & $98 \%$ & $100 \%$ & $64 \%$ \\
\hline $\mathrm{OH}$ & $100 \%$ & $0 \%$ & $0 \%$ & $2.799 \%$ & $100 \%$ & $100 \%$ & $25 \%$ \\
\hline OK & $20 \%$ & $0.101 \%$ & $0 \%$ & $0.864 \%$ & $97 \%$ & $100 \%$ & $59 \%$ \\
\hline OK & $100 \%$ & $0 \%$ & $0 \%$ & $1.29 \%$ & $100 \%$ & $100 \%$ & $21 \%$ \\
\hline PA & $20 \%$ & $0.086 \%$ & $0 \%$ & $0.937 \%$ & $94 \%$ & $100 \%$ & $63 \%$ \\
\hline PA & $100 \%$ & $0 \%$ & $0 \%$ & $2.749 \%$ & $100 \%$ & $100 \%$ & $15 \%$ \\
\hline RI & $20 \%$ & $0 \%$ & $0 \%$ & $1.646 \%$ & $100 \%$ & $100 \%$ & $54 \%$ \\
\hline RI & $100 \%$ & $0 \%$ & $0 \%$ & $0.711 \%$ & $100 \%$ & $100 \%$ & $69 \%$ \\
\hline $\mathrm{SC}$ & $20 \%$ & $0.01 \%$ & $0 \%$ & $0.193 \%$ & $99 \%$ & $100 \%$ & $83 \%$ \\
\hline $\mathrm{SC}$ & $100 \%$ & $0 \%$ & $0 \%$ & $1.233 \%$ & $100 \%$ & $100 \%$ & $50 \%$ \\
\hline SD & $20 \%$ & $0 \%$ & $0 \%$ & $0.66 \%$ & $99 \%$ & $100 \%$ & $71 \%$ \\
\hline SD & $100 \%$ & $0 \%$ & $0 \%$ & $1.312 \%$ & $100 \%$ & $100 \%$ & $27 \%$ \\
\hline
\end{tabular}


Table 4: Effect of the Number of Query Points.

\begin{tabular}{|c|c|c|c|c|c|c|c|}
\hline data & $N_{p}$ & $r(H W)$ & $r(H W 2)$ & $r(G D)$ & $o(H W)$ & $o(H W 2)$ & $o(G D)$ \\
\hline $\mathrm{CA}$ & 2 & $0 \%$ & $0 \%$ & $4.62 \%$ & $51 \%$ & $100 \%$ & $18 \%$ \\
\hline CA & 128 & $0 \%$ & $0 \%$ & $1.231 \%$ & $100 \%$ & $100 \%$ & $42 \%$ \\
\hline $\mathrm{OL}$ & 2 & $0 \%$ & $0 \%$ & $4.671 \%$ & $64 \%$ & $100 \%$ & $31 \%$ \\
\hline $\mathrm{OL}$ & 128 & $0 \%$ & $0 \%$ & $1.311 \%$ & $100 \%$ & $100 \%$ & $47 \%$ \\
\hline $\mathrm{AR}$ & 2 & $0 \%$ & $0 \%$ & $3.942 \%$ & $57 \%$ & $100 \%$ & $18 \%$ \\
\hline $\mathrm{AR}$ & 128 & $0 \%$ & $0 \%$ & $1.764 \%$ & $100 \%$ & $100 \%$ & $48 \%$ \\
\hline $\mathrm{AZ}$ & 2 & $0 \%$ & $0 \%$ & $5.112 \%$ & $56 \%$ & $100 \%$ & $15 \%$ \\
\hline $\mathrm{AZ}$ & 128 & $0 \%$ & $0 \%$ & $2.203 \%$ & $100 \%$ & $100 \%$ & $48 \%$ \\
\hline $\mathrm{CO}$ & 2 & $0 \%$ & $0 \%$ & $3.568 \%$ & $47 \%$ & $100 \%$ & $15 \%$ \\
\hline $\mathrm{CO}$ & 128 & $0 \%$ & $0 \%$ & $1.951 \%$ & $100 \%$ & $100 \%$ & $45 \%$ \\
\hline CT & 2 & $0 \%$ & $0 \%$ & $6.89 \%$ & $68 \%$ & $100 \%$ & $42 \%$ \\
\hline CT & 128 & $0.004 \%$ & $0 \%$ & $0.84 \%$ & $99 \%$ & $100 \%$ & $72 \%$ \\
\hline DE & 2 & $0 \%$ & $0 \%$ & $728 \%$ & $81 \%$ & $100 \%$ & $70 \%$ \\
\hline $\mathrm{DE}$ & 128 & $0 \%$ & $0 \%$ & $0.094 \%$ & $100 \%$ & $100 \%$ & $56 \%$ \\
\hline DR & 2 & $0 \%$ & $0 \%$ & $3.643 \%$ & $57 \%$ & $100 \%$ & $12 \%$ \\
\hline DR & 128 & $0 \%$ & $0 \%$ & $1.267 \%$ & $100 \%$ & $100 \%$ & $58 \%$ \\
\hline FL & 2 & $0 \%$ & $0 \%$ & $3.042 \%$ & $49 \%$ & $100 \%$ & $23 \%$ \\
\hline FL & 128 & $0.054 \%$ & $0 \%$ & $1.593 \%$ & $99 \%$ & $100 \%$ & $45 \%$ \\
\hline GA & 2 & $0 \%$ & $0 \%$ & $2.883 \%$ & $57 \%$ & $100 \%$ & $16 \%$ \\
\hline GA & 128 & $0 \%$ & $0 \%$ & $1.354 \%$ & $100 \%$ & $100 \%$ & $50 \%$ \\
\hline IA & 2 & $0 \%$ & $0 \%$ & $2.751 \%$ & $65 \%$ & $100 \%$ & $34 \%$ \\
\hline IA & 128 & $0 \%$ & $0 \%$ & $0.909 \%$ & $100 \%$ & $100 \%$ & $54 \%$ \\
\hline ID & 2 & $0 \%$ & $0 \%$ & $8.708 \%$ & $50 \%$ & $100 \%$ & $18 \%$ \\
\hline ID & 128 & $0.412 \%$ & $0 \%$ & $1.487 \%$ & $88 \%$ & $100 \%$ & $54 \%$ \\
\hline IL & 2 & $0 \%$ & $0 \%$ & $4.753 \%$ & $66 \%$ & $100 \%$ & $24 \%$ \\
\hline IL & 128 & $0 \%$ & $0 \%$ & $1.687 \%$ & $100 \%$ & $100 \%$ & $47 \%$ \\
\hline IN & 2 & $0 \%$ & $0 \%$ & $4.089 \%$ & $66 \%$ & $100 \%$ & $28 \%$ \\
\hline IN & 128 & $0 \%$ & $0 \%$ & $2.222 \%$ & $100 \%$ & $100 \%$ & $42 \%$ \\
\hline KS & 2 & $0 \%$ & $0 \%$ & $2.152 \%$ & $66 \%$ & $100 \%$ & $23 \%$ \\
\hline KS & 128 & $0 \%$ & $0 \%$ & $0.837 \%$ & $100 \%$ & $100 \%$ & $52 \%$ \\
\hline KY & 2 & $0 \%$ & $0 \%$ & $2.623 \%$ & $59 \%$ & $100 \%$ & $22 \%$ \\
\hline KY & 128 & $0.088 \%$ & $0 \%$ & $1.881 \%$ & $95 \%$ & $100 \%$ & $48 \%$ \\
\hline LA & 2 & $0 \%$ & $0 \%$ & $21.9 \%$ & $63 \%$ & $100 \%$ & $24 \%$ \\
\hline LA & 128 & $0.035 \%$ & $0 \%$ & $1.155 \%$ & $97 \%$ & $100 \%$ & $54 \%$ \\
\hline MA & 2 & $0 \%$ & $0 \%$ & $2.955 \%$ & $61 \%$ & $100 \%$ & $30 \%$ \\
\hline MA & 128 & $0.001 \%$ & $0 \%$ & $0.424 \%$ & $99 \%$ & $100 \%$ & $77 \%$ \\
\hline MD & 2 & $0 \%$ & $0 \%$ & $2.334 \%$ & $59 \%$ & $100 \%$ & $35 \%$ \\
\hline MD & 128 & $0 \%$ & $0 \%$ & $0.665 \%$ & $100 \%$ & $100 \%$ & $64 \%$ \\
\hline ME & 2 & $0 \%$ & $0 \%$ & $3.832 \%$ & $68 \%$ & $99 \%$ & $21 \%$ \\
\hline ME & 128 & $0 \%$ & $0 \%$ & $1.305 \%$ & $100 \%$ & $100 \%$ & $59 \%$ \\
\hline MI & 2 & $0 \%$ & $0 \%$ & $3.576 \%$ & $56 \%$ & $100 \%$ & $28 \%$ \\
\hline MI & 128 & $0.046 \%$ & $0 \%$ & $1.068 \%$ & $99 \%$ & $100 \%$ & $55 \%$ \\
\hline MN & 2 & $0 \%$ & $0 \%$ & $2.751 \%$ & $64 \%$ & $100 \%$ & $23 \%$ \\
\hline MN & 128 & $0.018 \%$ & $0 \%$ & $1.828 \%$ & $99 \%$ & $100 \%$ & $40 \%$ \\
\hline MO & 2 & $0 \%$ & $0 \%$ & $7.438 \%$ & $56 \%$ & $100 \%$ & $13 \%$ \\
\hline MO & 128 & $0 \%$ & $0 \%$ & $2.482 \%$ & $100 \%$ & $100 \%$ & $32 \%$ \\
\hline MS & 2 & $0 \%$ & $0 \%$ & $6.329 \%$ & $51 \%$ & $100 \%$ & $20 \%$ \\
\hline MS & 128 & $0 \%$ & $0 \%$ & $2.15 \%$ & $100 \%$ & $100 \%$ & $45 \%$ \\
\hline MT & 2 & $0 \%$ & $0 \%$ & $3.046 \%$ & $51 \%$ & $100 \%$ & $8 \%$ \\
\hline MT & 128 & $0 \%$ & $0 \%$ & $1.845 \%$ & $100 \%$ & $100 \%$ & $39 \%$ \\
\hline $\mathrm{NC}$ & 2 & $0 \%$ & $0 \%$ & $4.936 \%$ & $65 \%$ & $100 \%$ & $26 \%$ \\
\hline $\mathrm{NC}$ & 128 & $0.021 \%$ & $0 \%$ & $1.32 \%$ & $99 \%$ & $100 \%$ & $53 \%$ \\
\hline ND & 2 & $0 \%$ & $0 \%$ & $4.965 \%$ & $70 \%$ & $100 \%$ & $31 \%$ \\
\hline ND & 128 & $0 \%$ & $0 \%$ & $0.8 \%$ & $100 \%$ & $100 \%$ & $54 \%$ \\
\hline $\mathrm{NE}$ & 2 & $0 \%$ & $0 \%$ & $2.548 \%$ & $58 \%$ & $100 \%$ & $23 \%$ \\
\hline $\mathrm{NE}$ & 128 & $0 \%$ & $0 \%$ & $0.997 \%$ & $100 \%$ & $100 \%$ & $51 \%$ \\
\hline $\mathrm{NH}$ & 2 & $0 \%$ & $0 \%$ & $1.454 \%$ & $55 \%$ & $100 \%$ & $40 \%$ \\
\hline $\mathrm{NH}$ & 128 & $0 \%$ & $0 \%$ & $0.336 \%$ & $100 \%$ & $100 \%$ & $81 \%$ \\
\hline NJ & 2 & $0 \%$ & $0 \%$ & $3.946 \%$ & $71 \%$ & $100 \%$ & $42 \%$ \\
\hline NJ & 128 & $0 \%$ & $0 \%$ & $0.626 \%$ & $100 \%$ & $100 \%$ & $71 \%$ \\
\hline NM & 2 & $0 \%$ & $0 \%$ & $3.594 \%$ & $47 \%$ & $100 \%$ & $18 \%$ \\
\hline NM & 128 & $0 \%$ & $0 \%$ & $1.602 \%$ & $100 \%$ & $100 \%$ & $42 \%$ \\
\hline NV & 2 & $0 \%$ & $0 \%$ & $3.234 \%$ & $54 \%$ & $100 \%$ & $14 \%$ \\
\hline NV & 128 & $0 \%$ & $0 \%$ & $1.372 \%$ & $100 \%$ & $100 \%$ & $55 \%$ \\
\hline NY & 2 & $0 \%$ & $0 \%$ & $4.904 \%$ & $58 \%$ & $100 \%$ & $27 \%$ \\
\hline NY & 128 & $0.009 \%$ & $0 \%$ & $1.131 \%$ & $98 \%$ & $100 \%$ & $43 \%$ \\
\hline $\mathrm{OH}$ & 2 & $0 \%$ & $0 \%$ & $2.82 \%$ & $59 \%$ & $100 \%$ & $26 \%$ \\
\hline $\mathrm{OH}$ & 128 & $0 \%$ & $0 \%$ & $2.128 \%$ & $100 \%$ & $100 \%$ & $47 \%$ \\
\hline OK & 2 & $0 \%$ & $0 \%$ & $2.071 \%$ & $61 \%$ & $100 \%$ & $24 \%$ \\
\hline OK & 128 & $0 \%$ & $0 \%$ & $0.6 \%$ & $100 \%$ & $100 \%$ & $49 \%$ \\
\hline PA & 2 & $0 \%$ & $0 \%$ & $3.725 \%$ & $55 \%$ & $100 \%$ & $21 \%$ \\
\hline PA & 128 & $0 \%$ & $0 \%$ & $1.342 \%$ & $100 \%$ & $100 \%$ & $55 \%$ \\
\hline RI & 2 & $0 \%$ & $0 \%$ & $8.71 \%$ & $86 \%$ & $100 \%$ & $70 \%$ \\
\hline RI & 128 & $0 \%$ & $0 \%$ & $0.315 \%$ & $100 \%$ & $100 \%$ & $71 \%$ \\
\hline $\mathrm{SC}$ & 2 & $0 \%$ & $0 \%$ & $3.999 \%$ & $62 \%$ & $100 \%$ & $30 \%$ \\
\hline $\mathrm{SC}$ & 128 & $0 \%$ & $0 \%$ & $0.872 \%$ & $100 \%$ & $100 \%$ & $58 \%$ \\
\hline SD & 2 & $0 \%$ & $0 \%$ & $3.862 \%$ & $68 \%$ & $100 \%$ & $22 \%$ \\
\hline SD & 128 & $0 \%$ & $0 \%$ & $0.524 \%$ & $100 \%$ & $100 \%$ & $60 \%$ \\
\hline
\end{tabular}

Table 5: Effect of Multiple Windows.

\begin{tabular}{|c|c|c|c|c|c|c|c|}
\hline data & $\alpha$ & $r(H W)$ & $r(H W 2)$ & $r(G D)$ & $o(H W)$ & $o(H W 2)$ & $o(G D)$ \\
\hline $\mathrm{CA}$ & $20 \%$ & $0.023 \%$ & $0 \%$ & $2.974 \%$ & $91 \%$ & $100 \%$ & $17 \%$ \\
\hline CA & $40 \%$ & $0.012 \%$ & $0 \%$ & $2.615 \%$ & $98 \%$ & $100 \%$ & $24 \%$ \\
\hline $\mathrm{OL}$ & $20 \%$ & $0.23 \%$ & $0 \%$ & $2.594 \%$ & $82 \%$ & $100 \%$ & $31 \%$ \\
\hline $\mathrm{OL}$ & $40 \%$ & $0.028 \%$ & $0 \%$ & $1.583 \%$ & $98 \%$ & $100 \%$ & $26 \%$ \\
\hline $\mathrm{AR}$ & $20 \%$ & $0.033 \%$ & $0 \%$ & $1.789 \%$ & $94 \%$ & $100 \%$ & $34 \%$ \\
\hline AR & $40 \%$ & $0 \%$ & $0 \%$ & $1.41 \%$ & $100 \%$ & $100 \%$ & $45 \%$ \\
\hline $\mathrm{AZ}$ & $20 \%$ & $0.08 \%$ & $0 \%$ & $3.424 \%$ & $95 \%$ & $100 \%$ & $25 \%$ \\
\hline $\mathrm{AZ}$ & $40 \%$ & $0 \%$ & $0 \%$ & $2.088 \%$ & $100 \%$ & $100 \%$ & $34 \%$ \\
\hline $\mathrm{CO}$ & $20 \%$ & $0.01 \%$ & $0 \%$ & $3.246 \%$ & $95 \%$ & $100 \%$ & $18 \%$ \\
\hline $\mathrm{CO}$ & $40 \%$ & $0 \%$ & $0 \%$ & $2.42 \%$ & $100 \%$ & $100 \%$ & $34 \%$ \\
\hline CT & $20 \%$ & $0.321 \%$ & $0 \%$ & $1.819 \%$ & $70 \%$ & $100 \%$ & $37 \%$ \\
\hline CT & $40 \%$ & $0.201 \%$ & $0 \%$ & $2.103 \%$ & $89 \%$ & $100 \%$ & $59 \%$ \\
\hline DE & $20 \%$ & $0 \%$ & $0 \%$ & $0.103 \%$ & $93 \%$ & $100 \%$ & $64 \%$ \\
\hline DE & $40 \%$ & $0 \%$ & $0 \%$ & $0.164 \%$ & $100 \%$ & $100 \%$ & $70 \%$ \\
\hline DR & $20 \%$ & $0.017 \%$ & $0 \%$ & $2.154 \%$ & $94 \%$ & $100 \%$ & $33 \%$ \\
\hline DR & $40 \%$ & $0 \%$ & $0 \%$ & $2.245 \%$ & $100 \%$ & $100 \%$ & $42 \%$ \\
\hline FL & $20 \%$ & $0.237 \%$ & $0 \%$ & $2.95 \%$ & $70 \%$ & $100 \%$ & $20 \%$ \\
\hline FL & $40 \%$ & $0.13 \%$ & $0 \%$ & $2.531 \%$ & $85 \%$ & $100 \%$ & $19 \%$ \\
\hline GA & $20 \%$ & $0.058 \%$ & $0 \%$ & $2.554 \%$ & $95 \%$ & $100 \%$ & $31 \%$ \\
\hline GA & $40 \%$ & $0 \%$ & $0 \%$ & $1.989 \%$ & $100 \%$ & $100 \%$ & $42 \%$ \\
\hline IA & $20 \%$ & $0.026 \%$ & $0 \%$ & $1.876 \%$ & $95 \%$ & $100 \%$ & $26 \%$ \\
\hline IA & $40 \%$ & $0 \%$ & $0 \%$ & $0.925 \%$ & $100 \%$ & $100 \%$ & $40 \%$ \\
\hline ID & $20 \%$ & $0.335 \%$ & $0 \%$ & $3.103 \%$ & $63 \%$ & $100 \%$ & $19 \%$ \\
\hline ID & $40 \%$ & $0.448 \%$ & $0 \%$ & $3.011 \%$ & $81 \%$ & $100 \%$ & $29 \%$ \\
\hline IL & $20 \%$ & $0.067 \%$ & $0 \%$ & $2.188 \%$ & $96 \%$ & $100 \%$ & $23 \%$ \\
\hline IL & $40 \%$ & $0 \%$ & $0 \%$ & $2.217 \%$ & $100 \%$ & $100 \%$ & $28 \%$ \\
\hline IN & $20 \%$ & $0.038 \%$ & $0 \%$ & $1.887 \%$ & $95 \%$ & $100 \%$ & $28 \%$ \\
\hline IN & $40 \%$ & $0.002 \%$ & $0 \%$ & $1.76 \%$ & $99 \%$ & $100 \%$ & $35 \%$ \\
\hline KS & $20 \%$ & $0.022 \%$ & $0 \%$ & $1.506 \%$ & $93 \%$ & $100 \%$ & $19 \%$ \\
\hline $\mathrm{KS}$ & $40 \%$ & $0 \%$ & $0 \%$ & $1.001 \%$ & $100 \%$ & $100 \%$ & $37 \%$ \\
\hline KY & $20 \%$ & $0.223 \%$ & $0 \%$ & $2.915 \%$ & $85 \%$ & $100 \%$ & $34 \%$ \\
\hline KY & $40 \%$ & $0.019 \%$ & $0 \%$ & $2.033 \%$ & $99 \%$ & $100 \%$ & $42 \%$ \\
\hline LA & $20 \%$ & $0.212 \%$ & $0 \%$ & $3.598 \%$ & $68 \%$ & $100 \%$ & $22 \%$ \\
\hline LA & $40 \%$ & $0.041 \%$ & $0 \%$ & $2.312 \%$ & $92 \%$ & $100 \%$ & $38 \%$ \\
\hline MA & $20 \%$ & $0.097 \%$ & $0 \%$ & $1.42 \%$ & $84 \%$ & $100 \%$ & $28 \%$ \\
\hline MA & $40 \%$ & $0.013 \%$ & $0 \%$ & $1.736 \%$ & $99 \%$ & $100 \%$ & $43 \%$ \\
\hline MD & $20 \%$ & $0.221 \%$ & $0 \%$ & $1.263 \%$ & $83 \%$ & $100 \%$ & $41 \%$ \\
\hline MD & $40 \%$ & $0 \%$ & $0 \%$ & $2.04 \%$ & $100 \%$ & $100 \%$ & $51 \%$ \\
\hline ME & $20 \%$ & $0.062 \%$ & $0 \%$ & $2.597 \%$ & $88 \%$ & $100 \%$ & $28 \%$ \\
\hline ME & $40 \%$ & $0 \%$ & $0 \%$ & $1.485 \%$ & $100 \%$ & $100 \%$ & $48 \%$ \\
\hline MI & $20 \%$ & $0.191 \%$ & $0 \%$ & $5.801 \%$ & $65 \%$ & $100 \%$ & $24 \%$ \\
\hline MI & $40 \%$ & $0.226 \%$ & $0 \%$ & $3.97 \%$ & $78 \%$ & $100 \%$ & $28 \%$ \\
\hline MN & $20 \%$ & $0.039 \%$ & $0 \%$ & $1.956 \%$ & $94 \%$ & $100 \%$ & $22 \%$ \\
\hline MN & $40 \%$ & $0 \%$ & $0 \%$ & $1.709 \%$ & $100 \%$ & $100 \%$ & $36 \%$ \\
\hline MO & $20 \%$ & $0.046 \%$ & $0 \%$ & $3.844 \%$ & $87 \%$ & $100 \%$ & $12 \%$ \\
\hline MO & $40 \%$ & $0 \%$ & $0 \%$ & $3.094 \%$ & $100 \%$ & $100 \%$ & $24 \%$ \\
\hline MS & $20 \%$ & $0.034 \%$ & $0 \%$ & $2.742 \%$ & $95 \%$ & $100 \%$ & $22 \%$ \\
\hline MS & $40 \%$ & $0 \%$ & $0 \%$ & $2.212 \%$ & $100 \%$ & $100 \%$ & $38 \%$ \\
\hline MT & $20 \%$ & $0.063 \%$ & $0 \%$ & $2.582 \%$ & $90 \%$ & $100 \%$ & $19 \%$ \\
\hline MT & $40 \%$ & $0 \%$ & $0 \%$ & $2.178 \%$ & $100 \%$ & $100 \%$ & $27 \%$ \\
\hline $\mathrm{NC}$ & $20 \%$ & $0.13 \%$ & $0 \%$ & $2.842 \%$ & $82 \%$ & $100 \%$ & $18 \%$ \\
\hline $\mathrm{NC}$ & $40 \%$ & $0.06 \%$ & $0 \%$ & $2.195 \%$ & $96 \%$ & $100 \%$ & $42 \%$ \\
\hline ND & $20 \%$ & $0.024 \%$ & $0 \%$ & $1.684 \%$ & $94 \%$ & $100 \%$ & $30 \%$ \\
\hline ND & $40 \%$ & $0 \%$ & $0 \%$ & $1.083 \%$ & $100 \%$ & $100 \%$ & $44 \%$ \\
\hline $\mathrm{NE}$ & $20 \%$ & $0.019 \%$ & $0 \%$ & $2.185 \%$ & $97 \%$ & $100 \%$ & $21 \%$ \\
\hline $\mathrm{NE}$ & $40 \%$ & $0 \%$ & $0 \%$ & $1.53 \%$ & $100 \%$ & $100 \%$ & $40 \%$ \\
\hline $\mathrm{NH}$ & $20 \%$ & $0.131 \%$ & $0 \%$ & $2.838 \%$ & $86 \%$ & $100 \%$ & $32 \%$ \\
\hline $\mathrm{NH}$ & $40 \%$ & $0.016 \%$ & $0 \%$ & $1.699 \%$ & $98 \%$ & $100 \%$ & $50 \%$ \\
\hline NJ & $20 \%$ & $0.09 \%$ & $0 \%$ & $1.436 \%$ & $86 \%$ & $100 \%$ & $36 \%$ \\
\hline NJ & $40 \%$ & $0 \%$ & $0 \%$ & $2.208 \%$ & $100 \%$ & $100 \%$ & $45 \%$ \\
\hline NM & $20 \%$ & $0.047 \%$ & $0 \%$ & $2.632 \%$ & $95 \%$ & $100 \%$ & $18 \%$ \\
\hline NM & $40 \%$ & $0 \%$ & $0 \%$ & $2.052 \%$ & $100 \%$ & $100 \%$ & $31 \%$ \\
\hline NV & $20 \%$ & $0.031 \%$ & $0 \%$ & $2.689 \%$ & $92 \%$ & $100 \%$ & $21 \%$ \\
\hline NV & $40 \%$ & $0 \%$ & $0 \%$ & $2.146 \%$ & $100 \%$ & $100 \%$ & $48 \%$ \\
\hline NY & $20 \%$ & $0.014 \%$ & $0 \%$ & $2.324 \%$ & $93 \%$ & $100 \%$ & $25 \%$ \\
\hline NY & $40 \%$ & $0 \%$ & $0 \%$ & $1.457 \%$ & $100 \%$ & $100 \%$ & $34 \%$ \\
\hline $\mathrm{OH}$ & $20 \%$ & $0.045 \%$ & $0 \%$ & $2.919 \%$ & $90 \%$ & $100 \%$ & $23 \%$ \\
\hline $\mathrm{OH}$ & $40 \%$ & $0.006 \%$ & $0 \%$ & $3.057 \%$ & $99 \%$ & $100 \%$ & $28 \%$ \\
\hline OK & $20 \%$ & $0.026 \%$ & $0 \%$ & $1.648 \%$ & $90 \%$ & $100 \%$ & $33 \%$ \\
\hline $\mathrm{OK}$ & $40 \%$ & $0 \%$ & $0 \%$ & $1.204 \%$ & $100 \%$ & $100 \%$ & $32 \%$ \\
\hline PA & $20 \%$ & $0.134 \%$ & $0 \%$ & $2.301 \%$ & $87 \%$ & $100 \%$ & $28 \%$ \\
\hline PA & $40 \%$ & $0 \%$ & $0 \%$ & $2.032 \%$ & $99 \%$ & $100 \%$ & $33 \%$ \\
\hline RI & $20 \%$ & $0.078 \%$ & $0 \%$ & $1.078 \%$ & $88 \%$ & $100 \%$ & $62 \%$ \\
\hline RI & $40 \%$ & $0.007 \%$ & $0 \%$ & $0.474 \%$ & $98 \%$ & $100 \%$ & $63 \%$ \\
\hline SC & $20 \%$ & $0.023 \%$ & $0 \%$ & $2.615 \%$ & $94 \%$ & $100 \%$ & $29 \%$ \\
\hline SC & $40 \%$ & $0 \%$ & $0 \%$ & $1.059 \%$ & $100 \%$ & $100 \%$ & $53 \%$ \\
\hline SD & $20 \%$ & $0.005 \%$ & $0 \%$ & $1.056 \%$ & $97 \%$ & $100 \%$ & $36 \%$ \\
\hline SD & $40 \%$ & $0 \%$ & $0 \%$ & $1.459 \%$ & $100 \%$ & $100 \%$ & $39 \%$ \\
\hline
\end{tabular}

